\title{
Dark Matter from self-dual gauge/Higgs dynamics
}

\author{
Dario Buttazzo, ${ }^{a}$ Luca Di Luzio, ${ }^{a, b}$ Giacomo Landini, ${ }^{a, b}$ Alessandro Strumia ${ }^{b}$ \\ and Daniele Teresi ${ }^{a, b}$ \\ ${ }^{a}$ INFN, Sezione di Pisa, \\ Largo Bruno Pontecorvo 3, I-56127 Pisa, Italy \\ ${ }^{b}$ Dipartimento di Fisica "E. Fermi", Università di Pisa, \\ Largo Bruno Pontecorvo 3, I-56127 Pisa, Italy \\ E-mail: dario.buttazzo@pi.infn.it, luca.diluzio@pi.infn.it, \\ giacomo.landini@phd.unipi.it, alessandro.strumia@unipi.it, \\ daniele.teresi@df .unipi.it
}

ABSTRACT: We show that a new gauge group with one new scalar leads to automatically stable Dark Matter candidates. We consider theories where the Higgs phase is dual to the confined phase: it is known that $\mathrm{SU}(2)$ gauge theories with a scalar doublet (like the Standard Model) obey this non-trivial feature. We provide a general criterion, showing that this self-duality holds for $\mathrm{SU}(N), \mathrm{SO}(N), \mathrm{Sp}(N)$ and $G_{2}$ gauge dynamics with a scalar field in the fundamental representation. The resulting Dark Matter phenomenology has nontrivial features that are characteristic of the group, and that we discuss case by case. Just to mention a few, $\mathrm{SU}(N)$ has an accidental conserved dark baryon number, $\mathrm{SO}(2 N+1)$ leads to stable glue-balls thanks to a special parity, $G_{2}$ leads to a Dark Matter system analogous to neutral kaons. The cosmological Dark Matter abundance is often reproduced for masses around $100 \mathrm{TeV}$ : all constraints are satisfied and lighter dark glue-balls can affect Higgs physics. These theories acquire additional interest and predictivity assuming that both the dark and weak scales are dynamically generated.

KEYwords: Cosmology of Theories beyond the SM, Gauge Symmetry, Beyond Standard Model

ARXiv EPrint: 1907.11228 


\section{Contents}

1 Introduction 1

2 A fundamental of $\mathrm{SU}(\mathcal{N}) \quad 3$

2.1 SU: Higgs phase 3

2.2 SU: condensed phase 5

2.3 SU: phenomenology $\quad 7$

$\begin{array}{lll}\text { 2.3.1 Relic DM abundance } & 7\end{array}$

$\begin{array}{ll}2.3 .2 & \text { Dark glue-balls }\end{array}$

$\begin{array}{lll}2.3 .3 & \text { DM indirect detection } & 11\end{array}$

$\begin{array}{lll}2.3 .4 & \text { Direct detection } & 12\end{array}$

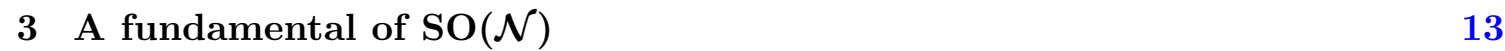

$\begin{array}{lll}3.1 & \text { SO: Higgs phase } & 13\end{array}$

$\begin{array}{lll}3.2 & \text { SO: condensed phase } & 14\end{array}$

$\begin{array}{lll}3.3 & \text { SO: phenomenology } & 15\end{array}$

$\begin{array}{lll}\text { 3.3.1 Relic DM abundance } & 15\end{array}$

$\begin{array}{lll}3.3 .2 & \text { DM indirect detection } & 17\end{array}$

$\begin{array}{lll}\text { 3.3.3 DM direct detection } & 17\end{array}$

$\begin{array}{lll}\text { 3.3.4 Special cases } & 18\end{array}$

4 A fundamental of $\operatorname{Sp}(\mathcal{N}) \quad 18$

$\begin{array}{lll}4.1 & \text { Sp: Higgs phase } & 18\end{array}$

$\begin{array}{lll}4.2 & \text { Sp: condensed phase } & 19\end{array}$

$\begin{array}{lll}4.3 & \text { Sp: phenomenology } & 20\end{array}$

4.3.1 Relic DM abundance 20

$\begin{array}{lll}4.3 .2 & \text { Indirect detection } & 20\end{array}$

4.3.3 Direct detection 20

5 A fundamental of $G_{2} \quad 20$

$5.1 G_{2}$ : Higgs phase 21

$5.2 G_{2}$ : condensed phase 22

$5.3 G_{2}$ : phenomenology 23

6 Conclusions 24

$\begin{array}{ll}\text { A Generators } & 27\end{array}$

$\begin{array}{lll}\text { A.1 } & \mathrm{SU}(\mathcal{N}) & 27\end{array}$

$\begin{array}{lll}\text { A.2 } & \mathrm{SO}(\mathcal{N}) & 27\end{array}$

$\begin{array}{lll}\text { A.3 } & \operatorname{Sp}(\mathcal{N}) & 27\end{array}$

A.4 $G_{2} \quad 28$

$\begin{array}{ll}\text { B Feynman rules } & 28\end{array}$ 


\section{Introduction}

We know that Dark Matter (DM) exists because we observed its collective gravitational interactions, but we do not know what DM is. Many theories are possible. Since gauge interactions are maximally predictive in relativistic quantum field theory, it makes sense to explore theories where gauge dynamics leads to DM. We thereby add a new 'dark' gauge group $\mathcal{G}$. Its glue-balls could be DM without any interaction with the Standard Model sector. In order to thermally reproduce the cosmological DM abundance we minimally connect the dark sector to the Standard Model by adding one scalar field $\mathcal{S}$ charged under $\mathcal{G}$. Depending on $\mathcal{G}$, this leads to non-trivial accidental symmetries that imply DM stability with non-standard physics. Despite that light elementary scalars are considered as unnatural by some theorists, interesting DM matter models based on scalars have already been proposed:

1) The most minimal DM model in terms of new degrees of freedom involves just one singlet scalar $\mathcal{S}[1-5]$. This is stable imposing an ad-hoc $\mathbb{Z}_{2}$ symmetry $\mathcal{S} \rightarrow-\mathcal{S}$ and assuming that the $\mathcal{S}$ vacuum expectation value vanishes. Direct detection bounds excluded a significant part of the parameter space of this model [1-5].

2) Next, if the field $\mathcal{S}$ is complex, describing two scalar degrees of freedom, it can be charged under a new $\mathcal{G}=\mathrm{U}(1)$ gauge group. A vacuum expectation value of $\mathcal{S}$ breaks $\mathrm{U}(1)$ to nothing and the resulting massive vector $A_{\mu}$ is a DM candidate, stable thanks to charge conjugation, $\mathcal{S} \rightarrow \mathcal{S}^{*}$ and $A_{\mu} \rightarrow-A_{\mu}$, which is a symmetry if the $\mathrm{U}(1)$ has vanishing kinetic mixing with hypercharge [6].

3) A more interesting model where DM stability is automatically implied by the particle content has been proposed in [7-9], assuming that the scalar $\mathcal{S}$ fills the fundamental representation 2 of a new $\mathrm{SU}(2)$ gauge group. A vacuum expectation value of $\mathcal{S}$ breaks $\mathrm{SU}(2)$ to nothing and the DM candidates are the three $\mathrm{SU}(2)$ vectors, which acquire a common mass because of an accidental custodial symmetry.

The SU(2) model admits two apparently different phases: Higgs and confined. A nontrivial feature of the $\mathrm{SU}(2)$ model - interesting even from a purely theoretical point of view - is that the two phases give the same spectrum of asymptotic particles. The lack of a sharp distinction between the Higgs and confined phases in $\mathrm{SU}(2)$ theories with a scalar in the fundamental has been proved by Fradkin, Shenker et al. [10-13]. A detailed analysis of how this surprising duality applies to the Standard Model can be found in [14, 15] (we now know that the $\mathrm{SU}(2)_{L}$ gauge group is weakly coupled, so that in the SM this duality has no physical interest).

We will find extra examples of Higgs/confinement dualities, and propose a general criterion: such a duality holds when a scalar $\mathcal{S}$ in a representation $R$ can break the gauge group $\mathcal{G}$ to a unique sub-group $\mathcal{H}$ (and thereby with a Higgs phase that is unique). In these cases $\mathcal{S}$ admits a single quartic coupling, and the broken theory contains a single Higgs scalar, that we call $s$. This happens when $\mathcal{S}$ fills a fundamental of the $\operatorname{SU}(\mathcal{N}), \operatorname{SO}(\mathcal{N})$, $\operatorname{Sp}(\mathcal{N}), G_{2}$ groups (up to equivalences). While in the original model [7-9] $\mathcal{G}=\mathrm{SU}(2)$ gets 
fully broken, in our examples $\mathcal{H}$ has a non-trivial gauge dynamics - its own confinement - that must be taken into account. On the other hand, a scalar in the fundamental of $F_{4}, E_{6}, E_{7}, E_{8}$, or in a higher representation of any group, such as a spinorial of $\mathrm{SO}(10)$, instead has multiple quartic couplings and gives inequivalent breaking patterns, leaving extra scalars in the broken theory.

We will here study theories that satisfy the Higgs/confinement duality, and their application to DM. Such theories can be seen as extensions of those previously listed in 1), $2), 3$ ), and give qualitatively new physics. We consider one elementary scalar $\mathcal{S}$ in the fundamental representation of a gauge group $\mathcal{G}$ with vectors $\mathcal{G}_{\mu \nu}^{a}$ in the adjoint. We consider the most generic renormalizable Lagrangian ${ }^{1}$

$$
\mathscr{L}=\mathscr{L}_{\mathrm{SM}}-\frac{1}{4} \mathcal{G}_{\mu \nu}^{a} \mathcal{G}^{a \mu \nu}-V_{\mathcal{S}}+ \begin{cases}\left|\mathcal{D}_{\mu} \mathcal{S}\right|^{2} & \text { if } \mathcal{S} \text { is complex }, \\ \left(\mathcal{D}_{\mu} \mathcal{S}\right)^{2} / 2 & \text { if } \mathcal{S} \text { is real }\end{cases}
$$

with scalar potential

$$
V_{\mathcal{S}}= \begin{cases}-M_{\mathcal{S}}^{2}|\mathcal{S}|^{2}+\lambda_{\mathcal{S}}|\mathcal{S}|^{4}-\lambda_{H \mathcal{S}}|H|^{2}|\mathcal{S}|^{2} & \text { if } \mathcal{S} \text { is complex } \\ -M_{\mathcal{S}}^{2} \mathcal{S}^{2} / 2+\lambda_{\mathcal{S}} \mathcal{S}^{4} / 4-\lambda_{H \mathcal{S}}|H|^{2} \mathcal{S}^{2} / 2 & \text { if } \mathcal{S} \text { is real. }\end{cases}
$$

$\mathcal{S}$ is complex when $\mathcal{G}=\mathrm{SU}(\mathcal{N})$ or $\operatorname{Sp}(\mathcal{N})$ : in such cases the theory is invariant under an accidental $\mathrm{U}(1)$ global symmetry, dark baryon number, that rotates the phase of $\mathcal{S}$. $\mathcal{S}$ is real when $\mathcal{G}=\operatorname{SO}(\mathcal{N})$ or $G_{2}$ : we will discuss the accidental symmetries of these theories. These minimal theories give non-trivial DM physics.

If $\mathcal{G}$ confines, baryons made of scalars $\mathcal{S}$ are stable DM candidates. As we will see, their nature qualitatively depends on the group $\mathcal{G}$. If $\mathcal{S}$ gets a vacuum expectation value, $\mathcal{G}$ gets broken to a subgroup $\mathcal{H}$,

$$
\mathrm{SU}(\mathcal{N}) \rightarrow \mathrm{SU}(\mathcal{N}-1), \quad \mathrm{SO}(\mathcal{N}) \rightarrow \mathrm{SO}(\mathcal{N}-1), \quad \mathrm{Sp}(\mathcal{N}) \rightarrow \mathrm{Sp}(\mathcal{N}-2), \quad G_{2} \rightarrow \mathrm{SU}(3),
$$

and some massive vectors are accidentally stable DM candidates. At lower energy $\mathcal{H}$ confines, giving rise to various states (dark glue-balls, dark mesons, ...) and to baryonic $\mathrm{DM}$, in such a way that the Higgs/confined and $\mathcal{G}$-confined phases are equivalent. ${ }^{2}$

Models with a new confining gauge group $\mathcal{G}$ and new fermions $\mathcal{F}$ have been explored in [19-21]: in such models communication with the SM arises if $\mathcal{F}$ is charged also under the SM gauge group: models need to be selected such that the composite DM is neutral.

\footnotetext{
${ }^{1}$ The dark gauge group can have an extra topological term. In the absence of fermions, it cannot be rotated away. Such term would violate $\mathrm{CP}$ at non-perturbative level. The $\mathrm{SU}(\mathcal{N}), \mathrm{SO}(2 \mathcal{N})$ and $E_{6}$ groups with symmetric Dynkin diagrams admit a $\mathbb{Z}_{2}$ outer automorphism (complex conjugation) [16] that acts on vectors by flipping the sign of some vectors, as determined by the vanishing of some $f^{a b c}$ group structure constants. More simply, the CP-even vectors are those associated to purely imaginary generators $T^{a}$ in some complex representation (e.g. fundamental or spinorial).

${ }^{2}$ In order to avoid confinement, $[17,18]$ considered non-minimal models with enough multiple scalars that $\mathrm{SU}(\mathcal{N})$ gets broken to nothing. We accept condensation and focus on the minimal scalar content. Given that DM is the lightest stable particle, this also approximates the DM phenomenology of more general theories provided that the extra particles are heavier at least by $\Delta M \gtrsim \Lambda_{\mathrm{DC}}$, where $\Lambda_{\mathrm{DC}}$ is the scale at which $g_{\mathrm{DC}}$ becomes strongly coupled, if unbroken.
} 
In scalar models, instead, we can assume that $\mathcal{S}$ is neutral under the SM (resulting into a neutral DM candidate) because $\mathcal{S}$ interacts with the Higgs through the mixed scalar quartic $\lambda_{H \mathcal{S}}$.

The paper is structured as follows. In section 2 we consider the group $\mathcal{G}=\operatorname{SU}(\mathcal{N})$ and study both the Higgs and condensed phases, focusing on their equivalence, on the accidental symmetry that protects the stability of DM, as seen by both the dual phases, and on DM phenomenology. We then extend the analysis to the other groups for which we find that the duality holds: $\operatorname{SO}(\mathcal{N})$ (section 3), $\operatorname{Sp}(\mathcal{N})$ (section 4 ) and $G_{2}$ (section 5). Conclusions are finally given in section 6 , where we summarize our main results.

\section{A fundamental of $\mathrm{SU}(\mathcal{N})$}

\subsection{SU: Higgs phase}

Independently of whether symmetry breaking happens dynamically, in the Higgs phase $\mathcal{S}$ can always be written as

$$
\mathcal{S}(x)=\frac{1}{\sqrt{2}}\left(\begin{array}{c}
0 \\
\vdots \\
0 \\
w+s(x)
\end{array}\right)
$$

such that the gauge group $\mathrm{SU}(\mathcal{N})$ gets broken to $\mathrm{SU}(\mathcal{N}-1)$, leaving one degree of freedom $s$ in $\mathcal{S}$. While $\langle\mathcal{S}\rangle$ breaks dark baryon number $\mathrm{U}(1)_{\mathrm{DB}}$ (under which $\mathcal{S}$ has charge 1), a stable DM candidate remains thanks to an accidental global U(1) symmetry. Its generator $\mathcal{N}(1, \ldots, 1,0) /(\mathcal{N}-1)$ is the unbroken linear combination of $\mathrm{U}(1)_{\mathrm{DB}}$ and the broken $\mathrm{U}(1)$ gauge symmetry in $\mathrm{SU}(\mathcal{N})$ corresponding to the generator (cf. appendix $\mathrm{A}$ )

$$
T^{\mathcal{N}^{2}-1}=\operatorname{diag}(1, \ldots, 1,1-\mathcal{N}) / \sqrt{2 \mathcal{N}(\mathcal{N}-1)} .
$$

Here and in the following, we normalize $\mathrm{SU}(\mathcal{N})$ generators in the fundamental representation as $\operatorname{Tr}\left(T^{a} T^{b}\right)=\frac{1}{2} \delta^{a b}$. It is especially interesting to consider dynamical symmetry breaking through the Coleman-Weinberg mechanism, obtained by setting $M_{\mathcal{S}}=0$. Assuming that $\lambda_{H \mathcal{S}}$ is negligibly small, the scalar $\mathcal{S}$ dynamically acquires a vacuum expectation value $w=s_{*} e^{-1 / 4}$ where $s_{*}$ is the Renormalization Group Equation (RGE) scale $\mu$ at which the running quartic coupling $\lambda_{\mathcal{S}}(\mu)$ crosses 0 , becoming negative at low energy, in view of its RGE at one loop

$$
\begin{aligned}
& (4 \pi)^{2} \frac{d g_{\mathrm{DC}}}{d \ln \mu}=-\frac{22 \mathcal{N}-1}{6} g_{\mathrm{DC}}^{3}, \\
& (4 \pi)^{2} \frac{d \lambda_{\mathcal{S}}}{d \ln \mu}=\frac{3}{4}(\mathcal{N}-1)\left(1+\frac{2}{\mathcal{N}}-\frac{2}{\mathcal{N}^{2}}\right) g_{\mathrm{DC}}^{4}-6 \mathcal{N} g_{\mathrm{DC}}^{2} \lambda_{\mathcal{S}}\left(1-\frac{1}{\mathcal{N}^{2}}\right)+4(4+\mathcal{N}) \lambda_{\mathcal{S}}^{2} .
\end{aligned}
$$

In such a case the scalar $s$ is known as 'scalon' [22] and its mass squared is one-loop suppressed, $M_{s}^{2}=w^{2} \beta_{\lambda_{S}}$, with $\beta_{\lambda_{S}} \equiv d \lambda_{S} / d \ln \mu$ [9]. If the Higgs mass term is absent too, this model can also generate the weak scale $v$, where $v \approx 246 \mathrm{GeV}$ is the needed Higgs 
vacuum expectation value. Assuming a small positive $\lambda_{H S}$, the weak scale is generated as $v \approx w \sqrt{\lambda_{H S} / 2 \lambda_{H}}$ [9]. More complicated expressions hold if $\lambda_{H S}$ is not negligibly small.

Writing the gauge bosons as

$$
T^{a} \mathcal{G}_{\mu}^{a}=\frac{1}{2}\left(\begin{array}{c|c}
\mathcal{A}_{\mu} & \sqrt{2} \mathcal{W}_{\mu} \\
\hline \sqrt{2} \mathcal{W}_{\mu}^{*} & 0
\end{array}\right)-\frac{\mathcal{Z}_{\mu}}{2} \sqrt{\frac{\mathcal{N}-1}{2 \mathcal{N}}}\left(\begin{array}{c|c}
-\mathbb{I} /(\mathcal{N}-1) & 0 \\
\hline 0 & 1
\end{array}\right)
$$

the perturbative spectrum is:

- the scalon $s$, singlet under $\mathrm{SU}(\mathcal{N}-1)$, with mass $M_{s}$;

- $\mathcal{N}(\mathcal{N}-2)$ massless dark gluons $\mathcal{A}_{\mu}$ in the adjoint of $\mathrm{SU}(\mathcal{N}-1)$;

- $2(\mathcal{N}-1)$ massive dark $\mathcal{W}_{\mu}$ in the $(\mathcal{N}-1)+\overline{(\mathcal{N}-1)}$ of $\mathrm{SU}(\mathcal{N}-1)$ with mass $M_{\mathcal{W}}^{2}=g_{\mathrm{DC}}^{2} w^{2} / 4$; the $\mathcal{W}$ are stable because charged under the global unbroken $\mathrm{U}(1)$;

- a massive dark $\mathcal{Z}_{\mu}$ corresponding to the generator $T^{\mathcal{N}^{2}-1}$ with mass $M_{\mathcal{Z}}^{2}=g_{\mathrm{DC}}^{2} w^{2}(\mathcal{N}-$ 1) $/ 2 \mathcal{N}$ that decays into $\mathcal{A A A}$ at one loop.

The case $\mathcal{N}=2$ of this model was studied in [7,8]: the dark gluons do not exist, and $\mathcal{W}, \mathcal{Z}$ are degenerate thanks to a custodial symmetry. We consider $\mathcal{N}>2$ such that $M_{\mathcal{W}}<$ $M_{\mathcal{Z}}<\sqrt{2} M_{\mathcal{W}}$. The DM candidate is the $\mathcal{W}$, that undergoes $\mathcal{W} \mathcal{W}^{*} \rightarrow \mathcal{A A}, s s, s \mathcal{A}, s \mathcal{Z}, \mathcal{A Z}$ annihilations, while co-annihilations $\mathcal{W Z} \rightarrow \mathcal{W} s[7]$ become irrelevant because $\mathcal{Z}$ is not $\mathrm{DM}$ for $\mathcal{N}>2$.

Condensation of $\mathbf{S U}(\mathcal{N}-\mathbf{1})$. The case $\mathcal{N}>2$ is qualitatively different from $\mathcal{N}=2$ because the vectors $\mathcal{A}$ confine at a scale $\Lambda_{\mathrm{DC}}$ that can be exponentially smaller than $M_{\mathcal{W}}$ :

$$
\Lambda_{\mathrm{DC}} \approx M_{\mathcal{W}} \exp \left[-\frac{6 \pi}{11(\mathcal{N}-1) \alpha_{\mathrm{DC}}\left(M_{\mathcal{W}}\right)}\right]
$$

where $\alpha_{\mathrm{DC}}\left(M_{\mathcal{W}}\right)$ is the value of the dark gauge coupling at the $\mathcal{W}$ mass. The squared masses of dark-colored particles receive extra contributions of order $\Lambda_{\mathrm{DC}}^{2}$. After the condensation of $\mathrm{SU}(\mathcal{N}-1)$, the spectrum of the theory contains:

- dark glue-balls $\mathcal{A A}$ with mass $M_{\mathrm{DG}} \sim 7 \Lambda_{\mathrm{DC}}[23]$;

- the dark scalon $s$;

- the $\mathcal{Z}$;

- scalar mesons $\mathcal{W W}^{*}$ that decay through the annihilation of their constituents;

- dark baryons $\mathcal{B} \sim \mathcal{W}^{\mathcal{N}-1}$, that remain as stable DM.

For $\Lambda_{\mathrm{DC}} \ll M_{\mathcal{W}}$ the dark baryon spectrum can be computed from non-relativistic quantum mechanics. ${ }^{3}$ In order to later address the condensed phase $\Lambda_{\mathrm{DC}} \sim M_{\mathcal{W}}$ (where the non-

\footnotetext{
${ }^{3}$ The lightest dark baryon is obtained by minimizing the angular momentum in the spatial part of its wave-function, compatibly with its symmetry under the exchange of two constituents. For a baryon the dark-color part is totally anti-symmetric, so the product of the spin and spatial wave-functions must also be totally anti-symmetric. For $\mathcal{N}=3,4$ the spin part alone can be anti-symmetrized, so that the ground state can have a totally symmetric $s$-wave. For higher values of $\mathcal{N}$, the spatial wave-function cannot be symmetric, and some orbital angular momentum must be involved.
} 
relativistic approximation does not hold) we here compute the dark baryon spectrum using a less usual formalism: by constructing gauge-singlet operators made of the constituent fields $\mathcal{W}_{\mu}^{I}$ and their covariant derivatives that interpolate between the dark baryon and the vacuum. For $\Lambda_{\mathrm{DC}} \ll M_{\mathcal{W}}$ we can keep the leading operator in the non-relativistic expansion in the velocity $\beta \sim p / M \sim \Lambda_{\mathrm{DC}} / M_{\mathcal{W}}$, under which the temporal index of vector fields as well as spatial derivatives are suppressed by $\beta$. Here we discuss the lowest values of $\mathcal{N}$ case by case:

$\diamond$ For $\mathcal{N}=3$ the lightest baryon is a vector because the anti-symmetric spin wavefunction is obtained as $(3 \otimes 3)_{\text {antisym }}=3$. Indeed, a combination at leading order in $\beta$ is $\mathcal{B}_{\mu}=\epsilon_{I J} \epsilon^{\mu \nu \lambda \rho} \mathcal{W}_{\nu}^{I} \mathcal{D}_{\lambda} \mathcal{W}_{\rho}^{J}$, which gives a spin-1 dark baryon. The leading spin-0 and spin-2 operators vanish because of anti-symmetry.

$\diamond$ For $\mathcal{N}=4$ the ground state is a scalar because the anti-symmetric spin wavefunction is obtained as $(3 \otimes 3 \otimes 3)_{\text {antisym }}=1$. The associated dark-baryon operator is, for instance, the scalar $\mathcal{B}=\epsilon_{I J K} \epsilon^{\mu \nu \rho \sigma} \mathcal{W}_{\mu}^{I} \mathcal{W}_{\nu}^{J}\left(\mathcal{D}_{\rho} \mathcal{W}_{\sigma}\right)^{K}$. The vector operator $\epsilon_{I J K} \epsilon^{\mu \nu \rho \sigma} \mathcal{W}_{\mu}^{I} \mathcal{W}_{\nu}^{J} \mathcal{W}_{\rho}^{K}$ excites a physical spin-1 resonance only at higher order in $\beta$.

$\diamond$ For $\mathcal{N}=5$ the lowest-lying bound state is a spin-1 resonance, corresponding for instance to the operator $\mathcal{B}_{\lambda}=\epsilon_{I J K L} \epsilon^{\mu \nu \rho \sigma}\left(\mathcal{D}_{\mu} \mathcal{W}_{\nu}\right)^{I} \mathcal{W}_{\rho}^{J} \mathcal{W}_{\sigma}^{K} \mathcal{W}_{\lambda}^{L}$. A scalar state arises at higher order in the non-relativistic limit, e.g. from the $\mathcal{O}(\beta)$ operator $\epsilon_{I J K L} \epsilon^{\mu \nu \rho \sigma} \mathcal{W}_{\mu}^{I} \mathcal{W}_{\nu}^{J} \mathcal{W}_{\rho}^{K} \mathcal{W}_{\sigma}^{L}$

$\diamond$ For $\mathcal{N}=6$ two derivatives are needed and at leading order the combination $\mathcal{B}_{\lambda}=\epsilon_{I J K L M} \epsilon^{\mu \nu \rho \sigma} \epsilon^{\lambda \tau \eta \xi} \mathcal{W}_{\mu}^{I} \mathcal{W}_{\nu}^{J} \mathcal{W}_{\rho}^{K}\left(\mathcal{D}_{\sigma} \mathcal{W}_{\tau}^{L}\right)\left(\mathcal{D}_{\eta} \mathcal{W}_{\xi}^{M}\right)$ is possible, which gives a spin-1 state.

Similar considerations can be done for higher $\mathcal{N}$. In each case, different resonances are split by an amount $\Delta M \sim \alpha_{\mathrm{DC}}^{2} M_{\mathcal{W}}$. For some values of $\mathcal{N}$, bound states with different spin exist at leading order; their fine-structure mass splitting is $\Delta M \sim \alpha_{\mathrm{DC}}^{4} M_{\mathcal{W}}$.

\subsection{SU: condensed phase}

In the discussion above, we studied the Higgs phase of a theory with a $\mathrm{SU}(\mathcal{N})$ gauge group and a scalar $\mathcal{S}$ in the fundamental representation. The same theory admits an apparently different confined phase, where the gauge group $\mathrm{SU}(\mathcal{N})$ becomes strong at energies $\Lambda_{\mathrm{DC}} \gtrsim M_{\mathcal{S}}$ (confinement happens before Higgsing) such that a $\left\langle\mathcal{S S}^{*}\right\rangle$ condensate forms, rather than a vacuum expectation value.

While the strong dynamics of scalars is mostly unknown, Fradkin et al. [10-13] claim a non-trivial theorem: in the presence of a scalar in the fundamental there is no sharp distinction between the confined phase and the Higgs phase. This means that the same asymptotic particles appear in the spectrum - a surprising result given that the two phases naively look different (for example, dark baryon number is unbroken in the confined phase). More in general, asymptotic states (even in the Higgs phase) should be described through gauge-invariant operators. This state/operator association has practical use in lattice computations and is interesting from a formal point of view as a way to describe 
physics in an explicitly gauge-invariant way, in particular avoiding splitting fields as a fluctuation over a vacuum expectation value.

The case with $\mathcal{N}=2$ has been explicitly discussed in $[8],{ }^{4}$ showing that both phases lead to a real scalar $s$ and to 3 degenerate massive vectors $\mathcal{Z}_{\mu}, \mathcal{W}_{\mu}, \mathcal{W}_{\mu}^{*}$. As discussed above, in the Higgs phase $s$ is the radial part of $\mathcal{S}$, and $\mathcal{W}_{\mu}, \mathcal{Z}_{\mu}$ are the vectors of $\mathrm{SU}(2)_{\mathrm{DC}}$. In the confined phase asymptotic states (bound states) are associated to gauge-invariant (singlet) operators. The same asymptotic states are recovered as [7]

$$
s=\mathcal{S S}^{\dagger}, \quad \mathcal{Z}_{\mu}=\mathcal{S}^{\dagger} \mathcal{D}_{\mu} \mathcal{S}, \quad \mathcal{W}_{\mu}=\mathcal{S} \mathcal{D}_{\mu} \mathcal{S}, \quad \mathcal{W}_{\mu}^{*}=\mathcal{S}^{\dagger} \mathcal{D}_{\mu} \mathcal{S}^{\dagger}
$$

with $M_{\mathcal{W}}=M_{\mathcal{Z}}$ dictated by a custodial symmetry. The $\mathcal{W}$ states involve contractions with the SU(2)-invariant $\epsilon_{i j}$ tensor and thereby are replaced by $S^{\mathcal{N}}$ baryons for $\mathcal{N}>2$. Indeed, $\mathcal{N}>2$ leads to a more complicated dynamics in the Higgs phase, due to the unbroken $\mathrm{SU}(\mathcal{N}-1)$ that confines at lower energy (the relevance of this confinement for the validity of the theorem was stressed in [24]). The main states found in the confined phase match those found in the Higgs phase as follows: ${ }^{5}$

- Glue-balls, associated to $\mathcal{G}_{\mu \nu}^{a} \mathcal{G}_{\mu \nu}^{a}$;

- The scalar $\mathcal{S}^{\dagger} \mathcal{S}$ corresponds to $s$;

- The vector $\mathcal{S}^{\dagger} \mathcal{D}_{\mu} \mathcal{S}$ corresponds to $\mathcal{Z}_{\mu}$;

- Baryon states $\mathcal{B} \sim \mathcal{S}^{\mathcal{N}}$ can be constructed as follows: in view of the contraction with the totally anti-symmetric tensor with $\mathcal{N}$ indices, a non-zero contribution is obtained when at least $\mathcal{N}-1$ terms contain covariant derivatives $\mathcal{D}$ acting on the corresponding $\mathcal{S}$; the baryonic interpolating operators can thus be written as

$$
\mathcal{B}=\mathcal{S}^{I}\left[\epsilon_{I J K \cdots}\left(\mathcal{D}^{(n)} \mathcal{S}\right)^{J}\left(\mathcal{D}^{\left(n^{\prime}\right)} \mathcal{S}\right)^{K} \cdots\right]
$$

To see that the condensed baryons $\mathcal{B} \sim \mathcal{S}^{\mathcal{N}}$ correspond to the baryons of the Higgs phase $\mathcal{B} \sim \mathcal{W}^{\mathcal{N}-1}$ discussed in section 2.1, we notice that when one component of $\mathcal{S}$ acquires a vacuum expectation value, the term in the square brackets in eq. (2.7) reduces to the $\mathcal{W}^{\mathcal{N}-1}$ baryons formed in the Higgs phase when the residual $\operatorname{SU}(\mathcal{N}-1)$ confines, after the identification of the $\mathcal{N}-1$ Goldstone bosons $\mathcal{D}_{\mu} \mathcal{S}^{J} \leftrightarrow \mathcal{W}_{\mu}^{J}$. The baryons of the Higgs and confined phases are in a one-to-one correspondence, consistently with Fradkin-Shenker theorem [10-13].

\footnotetext{
${ }^{4}$ The SM provides a more complicated example of a $\mathrm{SU}(2)$ theory with extra fermions: the equivalence of the Higgs and confined phases has been discussed in $[14,15]$.

${ }^{5} \mathrm{In}$ the case of $\mathrm{SU}(3)_{\mathrm{DC}}$ the validity of the theorem has been tested through lattice computations [25-27], with a puzzling result. Even in a weakly coupled theory the physics in the gauge-invariant formalism seems different from the physics found in the standard formalism, where scalars are split in a gauge dependent way as fluctuations over a vacuum expectation value. For $\mathcal{N}=2$ (for example, in the SM) the difference is claimed to be in details of cross sections; for $\mathcal{N}=3$ the difference is claimed to be already at the level of the spectrum of asymptotic states. We follow the standard procedure.
} 


\subsection{SU: phenomenology}

As the Higgs and confined phases contain the same asymptotic particles, we perform all computations in the Higgs phase for weak couplings.

The model has 4 extra parameters beyond the SM: $g_{\mathrm{DC}}, \lambda_{S}, \lambda_{H S}, M_{\mathcal{S}}^{2}$. For simplicity, we will discuss predictions in the dimension-less limit, where the model has only 2 extra parameters beyond the SM, given that $M_{\mathcal{S}}$ vanishes and that the weak scale $v$ is generated by the dynamical scale $w$, such that $\lambda_{H S}$ is fixed by

$$
v \simeq w \sqrt{\frac{\lambda_{H S}}{2 \lambda_{H}}}
$$

Furthermore, one can trade $\lambda_{\mathcal{S}}$ for the DM mass $(\mathcal{N}-1) M_{\mathcal{W}}$, and determine its value by assuming that the thermal DM relic abundance reproduces the observed cosmological abundance.

At the end, only $g_{\mathrm{DC}}$ remains as a free parameter. The condensed phase is smoothly obtained in the limit where $g_{\mathrm{DC}} \sim 4 \pi / \sqrt{\mathcal{N}-1}$ becomes non-perturbative. The phenomenology is similar to the $\mathcal{N}=2$ model of [9] with an important difference: the presence of extra light glue-balls.

\subsubsection{Relic DM abundance}

The thermal relic DM abundance is determined by various events.

First, the usual decoupling of free $\mathcal{W}$ vectors takes place at the temperature $T \sim M_{\mathcal{W}} / 25$. The relic abundance of $\mathcal{W}$ vectors is dictated by $\sigma v_{\text {rel }}$, the tree-level non-relativistic $s$ wave annihilation cross section (averaging over initial spin and gauge components, and multiplying by $\kappa=1 / 2$ for complex DM particles, or $\kappa=1$ for real DM particles). Using the Feynman rules collected in appendix B and summing over all annihilation processes we obtain the needed cross sections.

We first write those generic cross-sections that arise whenever DM vectors $\mathcal{W}$ with mass $M_{\mathcal{W}}$ fill a representation $R$ under the unbroken group $\mathcal{H}$ with massless vectors $\mathcal{A}$ :

$$
\begin{aligned}
\sigma v_{\text {rel }}\left(\mathcal{W} \mathcal{W}^{*} \rightarrow \mathcal{A A}\right) & =\kappa \frac{19 C_{R}\left(4 C_{R}-C_{\text {adj }}\right) g_{\mathrm{DC}}^{4}}{288 \pi d_{R} M_{\mathcal{W}}^{2}}, \\
\sigma v_{\text {rel }}\left(\mathcal{W W}^{*} \rightarrow \mathcal{A} s\right) & =\kappa \frac{2 C_{R} g_{\mathrm{DC}}^{2}}{9 \pi d_{R} w^{2}} \\
\sigma v_{\mathrm{rel}}\left(\mathcal{W} \mathcal{W}^{*} \rightarrow s s\right) & =\kappa \frac{11 M_{\mathcal{W}}^{2}}{144 \pi d_{R} w^{4}} .
\end{aligned}
$$

In the above expressions $d_{R}$ is the dimension of the representation $R, C_{R}$ is the quadratic Casimir of $R$ (defined as $\delta_{i j} C_{R}=\left(T_{R}^{a} T_{R}^{a}\right)_{i j}$ ), and $C_{\text {adj }}$ is the one of the adjoint of $\mathcal{H}$. Specializing to the case $\mathcal{H}=\mathrm{SU}(n)$ with $n=\mathcal{N}-1$, we have $C_{\text {adj }}=n, C_{\text {fund }}=\left(n^{2}-1\right) / 2 n$, 
$d_{\text {fund }}=n$, and $\kappa=1 / 2$ for the complex fundamental, so that ${ }^{6}$

$$
\begin{aligned}
\sigma v_{\text {rel }}\left(\mathcal{W} \mathcal{W}^{*} \rightarrow \mathcal{A A}\right) & =\frac{19 g_{\mathrm{DC}}^{4}\left(n^{2}-1\right)\left(n^{2}-2\right)}{1152 \pi n^{3} M_{\mathcal{W}}^{2}} \\
\sigma v_{\text {rel }}\left(\mathcal{W} \mathcal{W}^{*} \rightarrow \mathcal{A} s\right) & =\frac{g_{\mathrm{DC}}^{4}\left(n^{2}-1\right)}{72 \pi n^{2} M_{\mathcal{W}}^{2}} \\
\sigma v_{\text {rel }}\left(\mathcal{W} \mathcal{W}^{*} \rightarrow s s\right) & =\frac{11 g_{\mathrm{DC}}^{4}}{4608 \pi n M_{\mathcal{W}}^{2}} \\
\sigma v_{\text {rel }}\left(\mathcal{W W}^{*} \rightarrow \mathcal{Z} s\right) & =\frac{g_{\mathrm{DC}}^{4}(n+1)}{72 \pi n^{2} M_{\mathcal{W}}^{2}}\left(1-\frac{M_{\mathcal{Z}}^{2}}{4 M_{\mathcal{W}}^{2}}\right)^{3}, \\
\sigma v_{\text {rel }}\left(\mathcal{W} \mathcal{W}^{*} \rightarrow \mathcal{Z} \mathcal{A}\right) & =\frac{g_{\mathrm{DC}}^{4}(n-1)(n+1)^{2}}{288 \pi n^{3} M_{\mathcal{W}}^{2}}\left(1-\frac{M_{\mathcal{Z}}^{2}}{4 M_{\mathcal{W}}^{2}}\right)\left(19+\frac{M_{\mathcal{Z}}^{2}}{M_{\mathcal{W}}^{2}}+\frac{M_{\mathcal{Z}}^{4}}{4 M_{\mathcal{W}}^{4}}\right)
\end{aligned}
$$

where the last two extra cross sections involve the $\mathcal{Z}$ vector present in SU models. These cross sections are enhanced by order-one Sommerfeld and bound-state effects, that we neglect. If $\mathcal{W}$ decoupling were the only process, it would leave the present abundance

$$
\frac{\Omega_{\mathcal{W}} h^{2}}{0.110} \approx \frac{\sigma v_{\text {cosmo }}}{\sigma v_{\text {rel }}} \quad \text { where } \quad \sigma v_{\text {cosmo }} \approx 2.2 \times 10^{-26} \frac{\mathrm{cm}^{3}}{\mathrm{sec}} .
$$

Second, at the scale of dark confinement the $\mathcal{W}$ forms either mesons $\mathcal{W} \mathcal{W}^{*}$ that annihilate, or baryons $\mathcal{B} \sim \mathcal{W}^{\mathcal{N}-1}$ that remain as DM. The mass fraction in baryons is estimated as [20]

$$
\wp_{\mathcal{B}} \approx \frac{1}{1+2^{\mathcal{N}-2} /(\mathcal{N}-1)} .
$$

Third, the $\mathcal{B}$ baryons can annihilate with $\mathcal{B}^{*}$. The cross section is enhanced by recombination and ranges between the squared Bohr radius of the ground state, $\pi R_{\mathcal{B}}^{2}$ up to $1 / \Lambda_{\mathrm{DC}}^{2}$ depending on which levels get occupied during the cosmological evolution. If such annihilations happen at temperatures above the Coloumbian binding energy of these states, the cross section gets enhanced by the thermal size of the occupied levels [20, 28].

Fourth, if scalons $s$ and/or glue-balls $\mathcal{A A}$ produced by DM annihilations have a long enough life-time, so that they dominate the energy density of the Universe while decaying into SM particles, the reheating effects dilutes the DM density.

As physics is complex, it is useful to show estimates that exhibit the dependencies on parameters. The thermal relic DM abundance is estimated as $Y_{\mathrm{DM}} \equiv n_{\mathrm{DM}} / s \sim$ $1 /\left(T_{\mathrm{dec}} M_{\mathrm{Pl}} \sigma_{\mathrm{ann}}\right)$, by demanding $n_{\mathrm{DM}} \sigma \sim H$, where $H$ is the Hubble rate:

- Perturbative annihilations with $\sigma_{\mathrm{ann}} \sim \alpha_{\mathrm{DC}}^{2} / M_{\mathcal{W}}^{2}$ decouple at $T_{\mathrm{dec}} \sim M_{\mathcal{W}} / 25$ leaving $Y_{\mathrm{DM}} \sim M_{\mathcal{W}} / \alpha_{\mathrm{DC}}^{2} M_{\mathrm{Pl}}$.

\footnotetext{
${ }^{6}$ As a check of our computation, we verified that all cross sections scale as $1 / s$ in the ultra-relativistic limit, often thanks to cancellations related to the Higgs mechanism. The $\mathcal{W W}^{*} \rightarrow \mathcal{Z Z}$ process is kinematically closed. For $\mathcal{N}=2$ cross sections involving $\mathcal{A}$ vanish, $\mathcal{Z}$ becomes $\mathrm{DM}$ forming a degenerate triplet with $\mathcal{W}$, and the result in [9] is reproduced taking into account the extra cross sections $2 \sigma v_{\text {rel }}\left(\mathcal{W} \mathcal{W}^{*} \rightarrow s s\right)=\sigma v_{\text {rel }}(\mathcal{Z Z} \rightarrow s s)$ and $2 \sigma v_{\text {rel }}\left(\mathcal{W} \mathcal{W}^{*} \rightarrow \mathcal{Z} s\right)=\sigma v_{\text {rel }}(\mathcal{W Z} \rightarrow \mathcal{W} s)=3 g_{\text {DC }}^{4} / 128 \pi M_{\mathcal{W}}^{2}$
} 
- If all bound states have large cross section $\sigma_{\mathrm{ann}} \sim 1 / \Lambda_{\mathrm{DC}}^{2}$ at $T_{\mathrm{dec}} \sim \Lambda_{\mathrm{DC}}$, this phase leaves $Y_{\mathrm{DM}} \sim \Lambda_{\mathrm{DC}} / M_{\mathrm{Pl}}{ }^{7}$

- However, DM can survive forming heavy baryons with $\sigma_{\mathrm{ann}} \sim \pi R_{\mathcal{B}}^{2} \sim 1 / M_{\mathcal{W}}^{2} \alpha_{\mathrm{DC}}^{2}$, so that $Y_{\mathrm{DM}} \sim \alpha_{\mathrm{DC}}^{2} M_{\mathcal{W}}^{2} / \Lambda_{\mathrm{DC}} M_{\mathrm{Pl}}$.

- If the binding energy of heavy baryons $E_{B} \sim \alpha_{\mathrm{DC}}^{2} M_{\mathcal{W}}$ is smaller than $T \sim \Lambda_{\mathrm{DC}}$, thermally excited baryons acquire a radius $R_{\mathcal{B}} \sim T / \Lambda_{\mathrm{DC}}^{2}$ such that $\sigma_{\mathrm{ann}} \sim 1 / \Lambda_{\mathrm{DC}}^{2}$ at $T \sim \Lambda_{\mathrm{DC}}$.

Notice that the DM annihilation cross section predicted by cosmology (and relevant for DM indirect detection) depends on model parameters proportionally to the DM mass over the DM decoupling temperature: in the usual case this ratio is $\approx 25$ giving the usual value of $\sigma v_{\text {cosmo; }}$; a larger cross section arises in our case in the Higgs phase, where $T_{\mathrm{dec}} \sim \Lambda_{\mathrm{DC}} \ll M_{\mathcal{W}}$.

The predicted relic density is plotted in figure 1 as a function of the two free parameters $\Lambda_{\mathrm{DC}}$ and $M_{\mathcal{W}}$. In the red (green) regions the overall DM abundance turns out to be above (below) the cosmological value, which is reproduced on the boundary between them. We show the results both assuming a Bohr-like annihilation cross-section, as well as a fully nonperturbative $1 / \Lambda_{\mathrm{DC}}^{2}$ one. All in all, the observed cosmological abundance $\Omega_{\mathrm{DM}} h^{2}=0.11$ can be obtained for $\mathrm{DM}$ masses of $\approx 100 \mathrm{TeV}$ or higher.

\subsubsection{Dark glue-balls}

Dark Glue-balls (DG) decay through the $\lambda_{H S}$ coupling. Their life-time can be computed as follows. Since the $s$ mass is one-loop suppressed with respect to the $\mathcal{W}$ mass, the one-loop effective interaction between $s$ and light vectors $\mathcal{A}$ can be obtained from their one loop RGE-corrected kinetic term

$$
-\frac{1}{4}\left(\mathcal{A}_{\mu \nu}^{a}\right)^{2}\left[1-b_{\mathcal{W}} \frac{\alpha_{\mathrm{DC}}}{4 \pi} \ln \frac{M_{\mathcal{W}}^{2}}{\bar{\mu}^{2}}\right]
$$

where $b_{\mathcal{W}}=-\frac{11}{3}+\frac{1}{6}=-\frac{7}{2}$ is the jump in the RGE coefficient for $g_{\mathrm{DC}}$ due to $\mathcal{W}$ particles with field-dependent squared mass $M_{\mathcal{W}}^{2}=\frac{1}{4} g_{\text {DC }}^{2}(w+s)^{2}$. Expanding for $s \ll w$ gives the interaction

$$
\mathscr{L}_{\mathrm{eff}}^{s \mathcal{A} \mathcal{A}}=-\frac{7 \alpha_{\mathrm{DC}}}{16 \pi}\left(\mathcal{A}_{\mu \nu}^{a}\right)^{2}\left(\frac{s}{w}-\frac{s^{2}}{2 w^{2}}+\cdots\right)
$$

which results in various decays depending on the mass ordering. If DG are heavier than the weak scale, they decay into Higgs components as

$$
\Gamma\left(\mathrm{DG} \rightarrow s \rightarrow H H^{\dagger}=h h+Z Z+W W\right)=\frac{49 f_{\mathrm{DG}}^{2} \alpha_{\mathrm{DC}}^{2} \lambda_{H S}^{2}}{2048 \pi^{3} M_{\mathrm{DG}} M_{s}^{4}} \operatorname{Re} \sqrt{1-\frac{4 M_{h, W, Z}^{2}}{M_{\mathrm{DG}}^{2}}}
$$

\footnotetext{
${ }^{7}$ Such a geometric cross section among bound states leads to DM annihilations only if constituents deexcite towards the ground state before that the bound state gets broken by another collision. If $M_{\mathcal{W}} \gg \Lambda_{\mathrm{DC}}$ large angular momenta are involved, and the cross section gets suppressed by a factor roughly estimated as $\min \left(1, \alpha_{\mathrm{DC}}^{7 / 4} \Lambda_{\mathrm{DC}}^{5 / 2} / M_{\mathcal{W}}^{1 / 2} T^{2}\right)$ in [29], which becomes of order unity only at a temperature $T$ mildly below $\Lambda_{\mathrm{DC}}$. A more precise but more model-dependent study of such issues has been performed in [30].
} 
$\mathrm{SU}(3)$

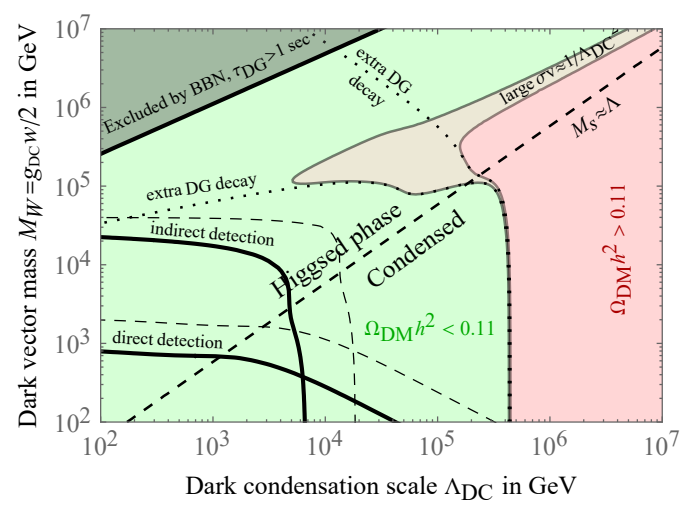

$\mathrm{SU}(10)$

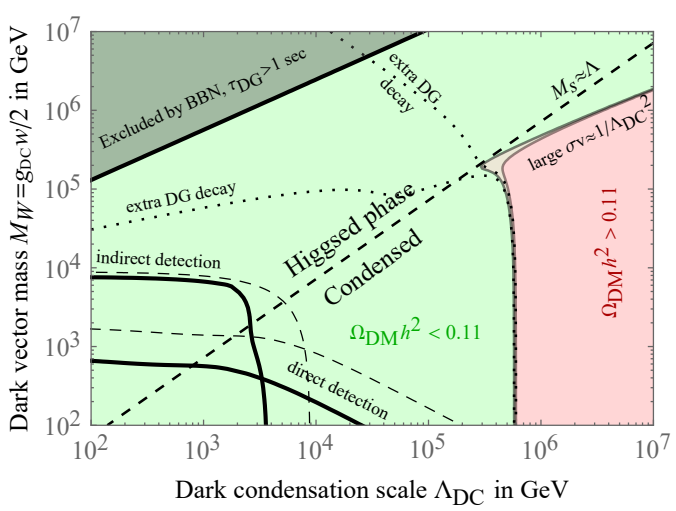

Figure 1. Physical quantities are here computed as function of the two main scale parameters, the dark color confinement scale $\Lambda_{\mathrm{DC}}$ and the constituent mass $M_{\mathcal{W}}=g_{\mathrm{DC}} w / 2$. The boundary between the Higgs and condensed phases $\left(g_{\mathrm{DC}} \sim 4 \pi / \sqrt{n}\right.$ i.e. $\left.\Lambda_{\mathrm{DC}} \sim M_{\mathcal{W}}\right)$ is shown as dashed line. The cosmological DM abundance is reproduced along the green/red boundaries, computed for two different values of non-perturbative DM annihilation at confinement: the (thermal) Bohr radius and $1 / \Lambda_{\mathrm{DC}}^{2}$. The latter possibility is relevant only if dark baryons do not fall cosmologically fast to their ground state. Numerical factors in the condensed phase are fixed matching to the perturbative cross-sections in the Higgs phase. We also show, as dotted curves, the same boundary computed assuming that some extra new physics gives fast glue-ball decays. The the upper gray region is excluded by BBN because of too slow DG decays and we also show limits from direct [31] and indirect [32] detection (plotted assuming the cosmological DM abundance). Future prospects are shown as dashed curves.

where $f_{\mathrm{DG}}=\left\langle 0\left|\left(\mathcal{A}_{\mu \nu}^{a}\right)^{2}\right| \mathrm{DG}\right\rangle \sim 3 M_{\mathrm{DG}}^{3} / g_{\mathrm{DC}}^{2} \sim M_{\mathrm{DG}}^{3}$ is a dark matrix element. The DG life-time can instead become cosmologically large if DG are enough lighter than the weak scale. If the scalon is heavy, integrating out $s$ gives the Higgs coupling to dark gluons

$$
\mathscr{L}_{\text {eff }}^{H \mathcal{A} \mathcal{A}}=-\frac{7 \alpha_{\mathrm{DC}} \lambda_{H S}}{16 \pi M_{s}^{2}}\left(H^{\dagger} H\right)\left(\mathcal{A}_{\mu \nu}^{a}\right)^{2},
$$

resulting in a mixing angle $\epsilon \ll 1$ between the Higgs and the DG, and in the consequent DG decay into SM particles

$$
\epsilon \approx \frac{7 \alpha_{\mathrm{DC}} \lambda_{H \mathcal{S}} v f_{\mathrm{DG}}}{16 \pi M_{s}^{2}\left(M_{h}^{2}-M_{\mathrm{DG}}^{2}\right)}, \quad \Gamma_{\mathrm{DG}} \approx \epsilon^{2} \Gamma_{h_{\mathrm{DG}}},
$$

where $\Gamma_{h_{\mathrm{DG}}}$ is the decay width of a SM Higgs with mass $M_{\mathrm{DG}}$. More in general, if $s$ can be as light as $h$, the above expression for $\epsilon$ gets replaced by

$$
\epsilon=\frac{7 \alpha_{\mathrm{DC}} f_{\mathrm{DG}}}{32 \pi w} \sin 2 \gamma\left(\frac{1}{M_{S_{1}}^{2}-M_{\mathrm{DG}}^{2}}-\frac{1}{M_{S_{2}}^{2}-M_{\mathrm{DG}}^{2}}\right)
$$

where $\gamma$ is the mixing angle that rotates the scalars $\{h, s\}$ to the mass-eigenstates $\left\{S_{1}, S_{2}\right\}$ with masses $M_{S_{1,2}}$. In terms of $v \approx 246 \mathrm{GeV}$ it is given by [9]

$$
\sin 2 \gamma=\frac{v^{2} \sqrt{8 \lambda_{H} \lambda_{H S}}}{M_{S_{2}}^{2}-M_{S_{1}}^{2}}
$$



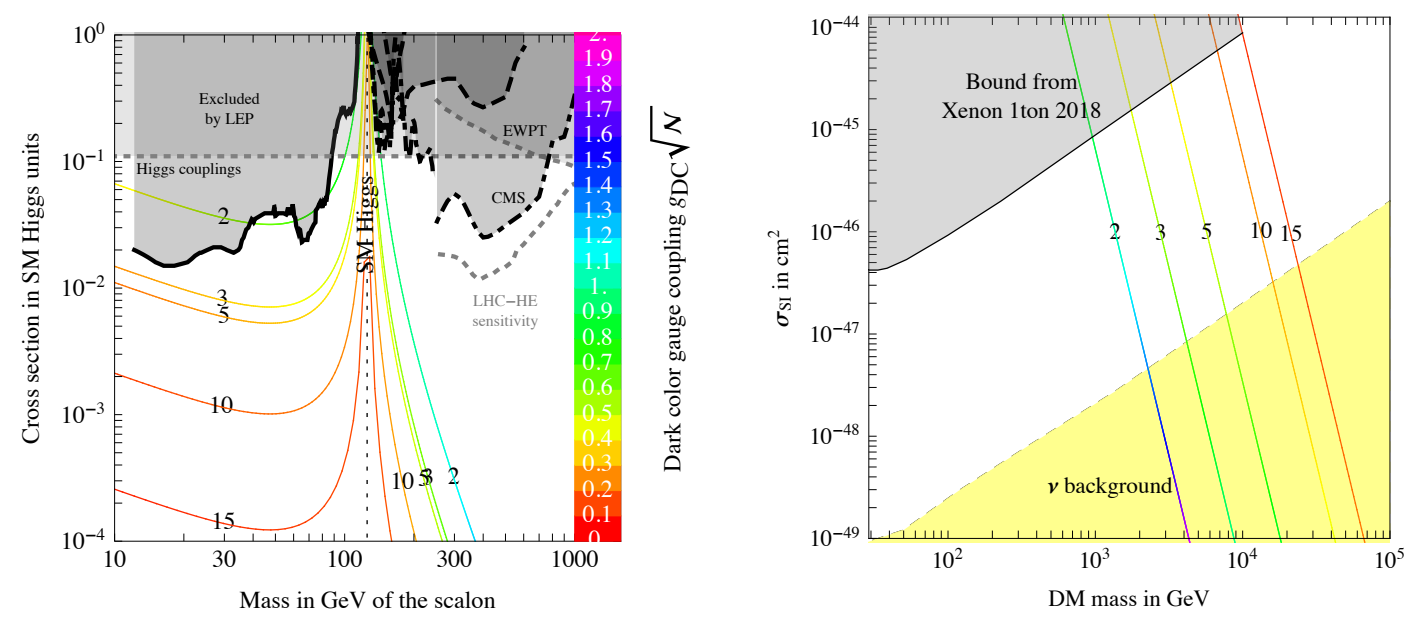

Figure 2. Predicted cross sections for the extra scalar boson (left) and for DM direct detection (right) as function of the only free parameter of the model $g_{\mathrm{DC}}$, varied as shown in the color legend. We here assume that DM has its cosmological abundance, and that it is negligibly affected by annihilations among dark baryons. The values of $\mathcal{N}$ are indicated on the curves.

with $M_{S_{2}}^{2} \approx 2 v^{2} \beta_{\lambda_{S}} \lambda_{H} / \lambda_{H S}$ and $M_{S_{1}}^{2} \approx 2\left(\lambda_{H}-\lambda_{H}^{2} / \beta_{\lambda_{S}}\right) v^{2}$, and where we fix $\lambda_{H}$ and $\lambda_{H S}$ in terms of the masses from eq. (2.3). In the limit $M_{S_{2}} \approx M_{s} \gg M_{h} \approx M_{S_{1}} \gg M_{\mathrm{DG}}$ the width reduces to eq. (2.17). The mixing angle turns out to be small under the assumptions of figure 1 for the relic density. In particular, the region where DM is a thermal relic gives signals much below present bounds. However the non-perturbative annihilation cross section among DM baryons might be smaller than what we assumed (see the discussion in footnote 7): sizeable signals arise in the opposite limit where annihilations among dark baryons negligibly suppress the DM abundance. In figure 2 we assume that the cosmological DM abundance is set by perturbative freeze-out and by the first recombination as in eq. (2.12). The left plot of figure 2 shows the predicted value of $\sin ^{2} \gamma$ in this case, which is equal to the production cross-section for the scalon $s$ in SM Higgs cross-section units (vertical axis), and of $M_{s}$ (horizontal axis) as function of $g_{\mathrm{DC}}$ (colored legend). Various present and projected constraints from Higgs measurements and direct searches are also shown [33, 34]. In particular, it can be seen that measuring Higgs couplings with a $10^{-3}$ precision, which can be attained at future lepton colliders, would allow to probe models where $s$ is light for several values of $\mathcal{N}$. Similar behaviours are found for the models discussed in the next sections.

\subsubsection{DM indirect detection}

The cross section for $\mathcal{B B}^{*}$ annihilations, that gives indirect detection signals, is enhanced by recombination. For small relative velocities $v_{\text {rel }}$, it can be written as

$$
\sigma_{\mathcal{B B}^{*}} v_{\mathrm{rel}} \approx \frac{\pi R_{\mathcal{B}}^{2}}{\sqrt{M_{\mathcal{B}} / 2 E_{\mathcal{B}}}}
$$


where $R_{\mathcal{B}}$ is the size of the baryon, $M_{\mathcal{B}}$ its mass, and $E_{\mathcal{B}}$ its binding energy. In the limit $\Lambda_{\mathrm{DC}} \ll M_{\mathcal{W}}$ where the constituents are non-relativistic one has

$$
R_{\mathcal{B}} \approx \frac{1}{C_{\text {fund }} \alpha_{\mathrm{DC}} M_{\mathcal{W}}}, \quad E_{\mathcal{B}} \approx n C_{\text {fund }}^{2} \alpha_{\mathrm{DC}}^{2} M_{\mathcal{W}},
$$

where we neglected factors of order one. Inserting the baryon mass $M_{\mathcal{B}} \approx n M_{\mathcal{W}}$ we get

$$
\sigma_{\mathcal{B B}} v_{\text {rel }} \approx \frac{\pi}{C_{\text {fund }} \alpha_{\mathrm{DC}} M_{\mathcal{W}}^{2}}
$$

For larger $\Lambda_{\mathrm{DC}} \gtrsim M_{\mathcal{W}}$ the constituents are no longer non-relativistic, and the cross-section becomes $\sigma_{\mathcal{B B}^{*}} \approx 1 / \Lambda_{\mathrm{DC}}^{2}$. Annihilations produce scalons and dark glue-balls, that decay into SM particles.

We compare the annihilation cross-section with the HESS limits on a gamma-ray signal in the galactic center [35]. The resulting bound, under the assumption that the dark baryons reproduce the full DM abundance of the Universe, is shown in figure 1 as a function of $\Lambda_{\mathrm{DC}}$ and $M_{\mathcal{W}}$. We also show future prospects at CTA [36]. However, in the region excluded by indirect detection the predicted DM abundance is much smaller than the cosmological abundance. The region where the DM abundance is reproduced thermally is allowed by bounds on indirect DM detection.

\subsubsection{Direct detection}

The DM dark baryon $\mathcal{B}$ couples to $s$ (and thereby to $h$ ) proportionally to its mass $M_{\mathcal{B}} \approx(\mathcal{N}-1) M_{\mathcal{W}}$ : the effective interaction is $2 M_{\mathcal{B}}^{2} s \mathcal{B}^{*} \mathcal{B} / w$. The resulting spin-independent cross section for direct detection is (both for $\mathcal{B}$ of spin 0 and spin 1 )

$$
\begin{aligned}
\sigma_{\mathrm{SI}} & =(\mathcal{N}-1)^{2} g_{\mathrm{DC}}^{2} \sin ^{2} 2 \gamma \frac{m_{N}^{4} f^{2}}{16 \pi v^{2}}\left(\frac{1}{M_{S_{1}}^{2}}-\frac{1}{M_{S_{2}}^{2}}\right)^{2} \\
& \approx 3.510^{-44} \mathrm{~cm}^{2} \times(\mathcal{N}-1)^{2} g_{\mathrm{DC}}^{2} \sin ^{2} 2 \gamma\left(1-\frac{M_{h}^{2}}{M_{S_{2}}^{2}}\right)^{2},
\end{aligned}
$$

where $f \approx 0.3$ is a nucleon matrix element. In the limit $M_{S_{2}} \approx M_{s} \gg M_{h}=M_{S_{1}}$ the cross section reduces to

$$
\sigma_{\mathrm{SI}} \approx(\mathcal{N}-1)^{2} \frac{m_{N}^{4} f^{2} v^{2} \lambda_{H} \lambda_{H S}}{2 \pi M_{h}^{4} M_{s}^{4}} g_{\mathrm{DC}}^{2}
$$

Figure 1 summarizes the situation as function of two parameters, $\Lambda_{\mathrm{DC}}$ and $M_{\mathcal{W}}$, without imposing that the cosmological DM abundance is reproduced. We show constraints from Xenon1T [31] as well as the neutrino floor, that limits future prospects. We again see that an allowed region exists, where all signals are significantly below present bounds. In particular the curve where the observed DM abundance is reproduced lies in the region allowed by all constraints. Here and below, we estimated that non-perturbativity arises for $g_{\mathrm{DC}} \approx 4 \pi / \sqrt{C_{\text {adj }}}$, equal to $4 \pi / \sqrt{n}$ for $\mathrm{SU}(\mathcal{N})$ models. At such value the Higgs phase smoothly becomes the confined phase. The DM mass that reproduces the cosmological density in the confined phase depends on uncertain strong dynamics. The right plot of figure 2 shows the predicted value of $\sigma_{\mathrm{SI}}$ (vertical axis) and of $M_{\mathcal{B}}$ (horizontal axis) as function 
of $g_{\mathrm{DC}}$ (colored legend) under the assumption that DM has its cosmological abundance and that it is negligibly suppressed by annihilations among DM baryons.

\section{A fundamental of $\mathrm{SO}(\mathcal{N})$}

The renormalizable Lagrangian is given in eq. (1.1), with $\mathcal{S}$ real (since a fundamental of $\mathrm{SO}(\mathcal{N})$ is a real representation). Thereby there is no accidentally conserved $\mathrm{U}(1)$ baryon number. We normalize the $\mathrm{SO}(\mathcal{N})$ generators in a non-standard way as $\operatorname{Tr}\left(T^{a} T^{b}\right)=2 \delta^{a b}$ in the fundamental and $\operatorname{Tr}\left(T^{a} T^{b}\right)=(2 \mathcal{N}-4) \delta^{a b}$ in the adjoint, in order to keep $\mathrm{SU}(2) \sim \mathrm{SO}(3)$ manifest. Since the $\mathrm{SO}(\mathcal{N})$ adjoint is the two-index anti-symmetric representation, the $\mathrm{SO}(\mathcal{N})$ gauge vectors $\mathcal{G}^{a}$ can be written as $\mathcal{G}_{i j}=\mathcal{G}^{a} T_{i j}^{a}$, anti-symmetric under $i \leftrightarrow j$. The RGE are

$$
\begin{aligned}
& (4 \pi)^{2} \frac{d g_{\mathrm{DC}}}{d \ln \mu}=-\frac{22 \mathcal{N}-45}{3} g_{\mathrm{DC}}^{3} \\
& (4 \pi)^{2} \frac{d \lambda_{\mathcal{S}}}{d \ln \mu}=6(\mathcal{N}-1) g_{\mathrm{DC}}^{4}-12(\mathcal{N}-1) g_{\mathrm{DC}}^{2} \lambda_{\mathcal{S}}+2(8+\mathcal{N}) \lambda_{\mathcal{S}}^{2}
\end{aligned}
$$

\subsection{SO: Higgs phase}

The most generic vacuum expectation value of $\mathcal{S}$ can be rotated to its $\mathcal{N}$-th component

$$
\mathcal{S}(x)=\left(\begin{array}{c}
0 \\
\vdots \\
0 \\
w+s(x)
\end{array}\right)
$$

such that $\mathrm{SO}(\mathcal{N})$ is broken to $\mathrm{SO}(\mathcal{N}-1)$. Writing the gauge bosons as

$$
T^{a} \mathcal{G}_{\mu}^{a}=i\left(\begin{array}{c|c}
\mathcal{A}_{\mu} & -\mathcal{W}_{\mu} \\
\hline \mathcal{W}_{\mu}^{T} & 0
\end{array}\right)
$$

the perturbative spectrum is:

- the singlet scalon $s$ with squared mass $M_{s}^{2}=w^{2} \beta_{\lambda_{S}}$;

- $(\mathcal{N}-1)(\mathcal{N}-2) / 2$ massless dark gluons $\mathcal{A}_{\mu}$ in the adjoint of $\operatorname{SO}(\mathcal{N}-1)$;

- $\mathcal{N}-1$ real $\mathcal{W}$ vectors in the fundamental of $\mathrm{SO}(\mathcal{N}-1)$ with mass $M_{\mathcal{W}}=g_{\mathrm{DC}} w$.

The vectors $\mathcal{W}$ are stable because the action is invariant under the $\operatorname{diag}(-1, \ldots,-1,1)$ $\mathrm{O}(\mathcal{N})$ reflection that leaves the $\mathcal{N}$-th component of $\mathcal{S}$ invariant, flipping all other components. In the broken theory this symmetry acts as

$$
s \rightarrow s, \quad \mathcal{W}_{\mu} \rightarrow-\mathcal{W}_{\mu}, \quad \mathcal{A}_{\mu} \rightarrow \mathcal{A}_{\mu}
$$

For $\mathrm{SO}(2)=\mathrm{U}(1)$ dark gluons $\mathcal{A}$ are absent and this symmetry reduces to $\mathrm{U}(1)$ charge conjugation. 
Condensation of $\operatorname{SO}(\mathcal{N}-1)$. When $\operatorname{SO}(\mathcal{N}-1)$ confines, DM forms dark mesons $\mathcal{W}_{i} \mathcal{W}_{i}$ (which annihilate), dark glue-balls $\mathcal{A} \mathcal{A}$ (which decay to SM particles), and various baryons, defined as states formed contracting one $\epsilon_{i_{1} \cdots i_{\mathcal{N}-1}}$ tensor with the fields of the theory: the heavy $\mathcal{W}_{i}^{\mu}$ and the light $\mathcal{A}_{i j}^{\mu \nu}$. The possibility of using dark gluons as valence constituents of baryons makes a qualitative difference with respect to the $\mathrm{SU}(\mathcal{N})$ case. The lightest baryon is the state that contains the lowest possible number of heavy $\mathcal{W}$ and, as discussed in the next sub-section, it is a stable DM candidate:

- for $\mathcal{N}$ even, baryons contain an odd number of $\mathcal{W}$, and the lightest baryon contains one $\mathcal{W}$ :

$$
\mathcal{B} \sim \mathcal{W}^{i_{1}} \mathcal{A}^{i_{2} i_{3}} \ldots \mathcal{A}^{i_{n-1} i_{n}} \epsilon_{i_{1} \cdots i_{n}}
$$

- for $\mathcal{N}$ odd, baryons contain an even number of $\mathcal{W}$, and the lightest baryon contains zero $\mathcal{W}$ :

$$
\mathcal{B} \sim \mathcal{A}^{i_{1} i_{2}} \ldots \mathcal{A}^{i_{n-1} i_{n}} \epsilon_{i_{1} \cdots i_{n}}
$$

\subsection{SO: condensed phase}

We next consider the phase where $g_{\mathrm{DC}}$ is non-perturbatively large, such that $\mathrm{SO}(\mathcal{N})$ condenses forming the following singlets under $\mathrm{SO}(\mathcal{N})$ :

- a meson $\mathcal{S}_{i} \mathcal{S}_{j} \delta_{i j}$, which is identified with the scalon $s$ in the Higgs phase; ${ }^{8}$

- glue-balls $\mathcal{G G}$, identified with the $\mathcal{A A}$ glue-balls in the Higgs phase;

- baryons formed with one $\epsilon_{i_{1} \cdots i_{\mathcal{N}}}$ tensor.

Differently from $\mathrm{SU}(\mathcal{N})$ baryons, the lightest $\mathrm{SO}(\mathcal{N})$ baryon does not need to be made of $\mathcal{N}$ fundamentals as $\epsilon_{i_{1} \cdots i_{\mathcal{N}}} \mathcal{S}_{i_{1}} \cdots \mathcal{S}_{i_{\mathcal{N}}}$. Rather, gauge bosons $\mathcal{G}_{i j}$ can be used to form $\operatorname{SO}(\mathcal{N})$ baryons. Two constituents $\mathcal{S}_{i} \mathcal{S}_{j}$ can annihilate into one dark gluon $\mathcal{G}_{i j}$. The lightest baryon presumably contains the minimal number of $\mathcal{S}$, zero or one:

$$
\begin{aligned}
\mathcal{B} \sim \mathcal{S}_{i_{1}} \mathcal{G}_{i_{2} i_{3}} \cdots \mathcal{G}_{i_{\mathcal{N}-1} i_{\mathcal{N}}} \epsilon_{i_{1} \cdots i_{\mathcal{N}}} & \text { for } \mathcal{N} \text { odd }, \\
\mathcal{B} \sim \mathcal{G}_{i_{1} i_{2}} \cdots \mathcal{G}_{i_{\mathcal{N}-1} i_{\mathcal{N}}} \epsilon_{i_{1} \cdots i_{\mathcal{N}}} & \text { for } \mathcal{N} \text { even, } \mathcal{B}^{2} \propto \operatorname{det} \mathcal{G} .
\end{aligned}
$$

These lightest baryons are in one-to-one correspondence with the ones in the broken phase. For $\mathcal{N}$ odd, when $\mathcal{S}$ gets a vev along its $\mathcal{N}$-th component, the remaining $\mathcal{G}$ constituents are identified with the $\mathcal{A}$ 's; for $\mathcal{N}$ even, exactly one of the $\mathcal{G}$ constituents is identified with the $\mathcal{W}$ in the broken phase due to the $\epsilon$ tensor, while the others are unbroken generators.

The lightest dark baryon is stable because the theory is accidentally invariant under $\mathrm{O}(\mathcal{N})$ rotations $R_{i j}$ with determinant -1 [37]. Acting on baryons $\mathcal{B}$ that contain the $\epsilon$ tensor, such rotations give $\mathcal{B} \rightarrow(\operatorname{det} R) \mathcal{B}$, since $R_{i_{1} j_{1}} \cdots R_{i_{\mathcal{N}} j_{\mathcal{N}}} \epsilon_{i_{1} \cdots i_{\mathcal{N}}}=\epsilon_{j_{1} \cdots j_{\mathcal{N}}} \operatorname{det} R$. After dividing by $\mathrm{SO}(\mathcal{N})$ rotations one gets a $\mathbb{Z}_{2}$ symmetry, that we dub O-parity. Dark baryons built with one $\mathcal{N}$-index anti-symmetric tensor are odd under this $\mathbb{Z}_{2}$ symmetry.

\footnotetext{
${ }^{8}$ Note that the gauge part in the operator $\mathcal{S}^{T} \mathcal{D}_{\mu} S$ disappears, because of the antisymmetry of the $\operatorname{SO}(\mathcal{N})$ generators. This corresponds to the absence of its corresponding $\mathcal{Z}$ boson in the Higgs phase.
} 
O-parity is a special unusual symmetry, analogous to space parity and time inversion, in the sense that it can be written in equivalent explicit ways only after choosing an arbitrary basis in the field space, thus fixing one arbitrary rotation with determinant -1 .

For $\mathcal{N}$ odd, O-parity is more conveniently realised as a full reflection $-\mathbb{I}$ i.e. O-parity acts as a usual $\mathbb{Z}_{2}$ symmetry

$$
\mathcal{S}_{i} \stackrel{\mathrm{O}}{\longrightarrow}-\mathcal{S}_{i}, \quad \mathcal{G}_{i j} \stackrel{\mathrm{O}}{\longrightarrow} \mathcal{G}_{i j}
$$

and the lightest baryon is stable because made of an odd number of $\mathcal{S}$ 's.

For $\mathcal{N}$ even, O-parity can be conveniently realised as a reflection under any direction, for example along the first component: $\eta_{1}=\operatorname{diag}(-1,1, \ldots, 1) \cdot{ }^{9}$ As any vector can be gauge-rotated to be along the 1 direction, this morally is parity. As $\eta_{1}$ anti-commutes with the generators $T^{1 i}$ (rotation along the $1 i$ plane) and commutes with the other generators, the Lagrangian is invariant under

$$
\mathcal{S}_{1} \stackrel{\mathrm{O}}{\longrightarrow}-\mathcal{S}_{1}, \quad \mathcal{S}_{i} \stackrel{\mathrm{O}}{\longrightarrow} \mathcal{S}_{i}, \quad \mathcal{G}^{1 i} \stackrel{\mathrm{O}}{\longrightarrow}-\mathcal{G}^{1 i}, \quad \mathcal{G}^{i j} \stackrel{\mathrm{O}}{\longrightarrow} \mathcal{G}^{i j}, \quad i, j \neq 1 .
$$

Two baryons can annihilate. The meson $\mathcal{S} \mathcal{S}$ and the glue-balls $\mathcal{G G}$ are even under O-parity and can decay into SM particles in view of the $\lambda_{H S}$ coupling.

For even $\mathcal{N}=2 N$ the theory contains an extra accidental symmetry different from O-parity. Indeed $\mathrm{SO}(2 N)$ admits one outer automorphism, which corresponds to complex conjugation $\mathrm{C}$. Its action on vector bosons can be computed by looking at the generators in the simplest complex representation, the spinor. $\mathrm{C}$ acts as

$$
\mathcal{G}_{i j} \stackrel{\mathrm{C}}{\longrightarrow}(-1)^{i+j} \mathcal{G}_{i j}, \quad \mathcal{S}_{i} \stackrel{\mathrm{C}}{\longrightarrow}(-1)^{i+1} \mathcal{S}_{i} .
$$

The consistency with the Lie algebra can be proved analogously to footnote 9 . Since $\mathcal{S}_{i} \mathcal{S}_{j}$ transforms as $\mathcal{G}_{i j}$ the $\mathrm{C}$ symmetry does not give extra stable baryons (despite the fact that for $\mathrm{SO}(4 N+2)$ the 0 -baryon is odd under $\mathrm{C}$, whereas for $\mathrm{SO}(4 N)$ it is even). ${ }^{10}$

\subsection{SO: phenomenology}

\subsubsection{Relic DM abundance}

As in $\mathrm{SU}(\mathcal{N})$ models, the thermal relic DM abundance is determined by various cosmological events.

\footnotetext{
${ }^{9}$ One can explicitly verify that this symmetry is consistent with the Lie algebra of $\operatorname{SO}(\mathcal{N})$
}

$$
\left[T^{a b}, T^{c d}\right]=i \delta^{a c} T^{b d}+\text { permutations }
$$

since: if no indices are 1 all generators appearing are even; if one index is 1 both sides are odd; if two indices are 1 both generators on the l.h.s. are odd and the r.h.s. is even.

${ }^{10}$ It might seem surprising that $\mathrm{C}$ acts non-trivially on $\mathcal{S}$ (a real representation). This becomes intuitive for $\mathrm{SO}(2)=\mathrm{U}(1)$ : two real scalars are seen as one complex scalar $\mathcal{S}_{1}+i \mathcal{S}_{2}$. For larger $N$, the C symmetry similarly reduces to the usual charge conjugation within the $\mathrm{SU}(N)$ subgroup of $\mathrm{SO}(2 N)$. Indeed, since $\mathrm{U}(N)=\mathrm{SO}(2 N) \cap \mathrm{Sp}(2 N)$, the $\mathrm{SU}(N)$ subalgebra of $\mathrm{SO}(2 N)$ has the form of eq. (A.5), with $\sigma_{k}=\sigma_{2}$. Since $\mathbb{I}_{2}$ is diagonal, the first set in eq. (A.5) is even under C of $\mathrm{SO}(2 N)$; since $T_{\alpha \beta}^{(2)}$ is imaginary, this is even also under $\mathrm{C}$ of $\mathrm{SU}(N): T_{\text {imag }} \rightarrow T_{\text {imag }}, T_{\text {real }} \rightarrow-T_{\text {real }}$. Analogously for the remaining two sets in eq. (A.5), since $\sigma_{2}$ is off-diagonal and $T_{\alpha \beta}^{(1)}$ is real. 
$\mathrm{SO}(4)$

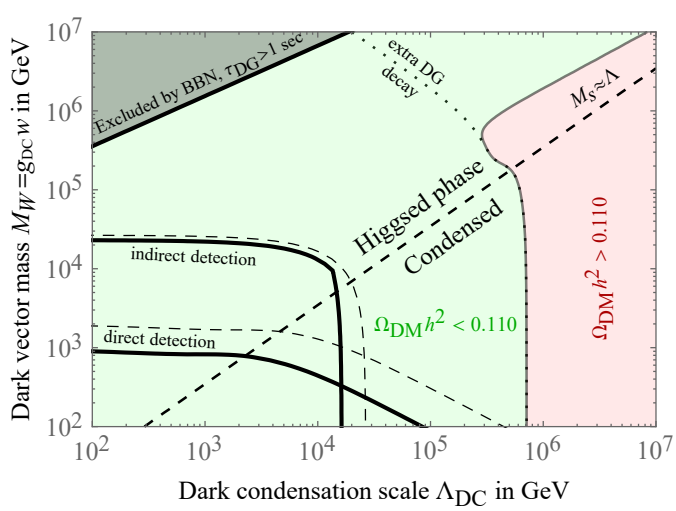

$\mathrm{SO}(5)$

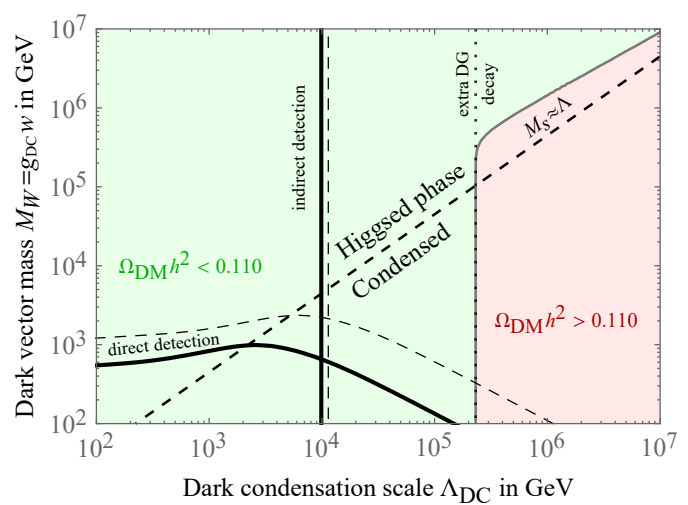

Figure 3. As in figure 1, for $\mathrm{SO}(\mathcal{N})$ models. The left plot shows an example with $\mathcal{N}$ even $(\mathrm{DM}$ is the baryon with one $\mathcal{W}$ constituent). The right plot shows an example with $\mathcal{N}$ odd (DM is the baryon with zero $\mathcal{W}$ constituents). In the right panel, the non-perturbative DM annihilation cross-section cannot be matched to its perturbative value: we assume $\sigma_{\mathrm{ann}} \approx 100 / \Lambda_{\mathrm{DC}}^{2}$.

Specializing eq. (2.9) to the unbroken $\mathcal{H}=\mathrm{SO}(n)$ group, so that $\kappa=1, d_{\text {fund }}=n=$ $\mathcal{N}-1, C_{\text {fund }}=n-1$ and $C_{\text {adj }}=2(n-2)$ in our normalization, we obtain the annihilation cross sections relevant for the usual decoupling of free $\mathcal{W}$ vectors

$$
\begin{gathered}
\sigma v_{\text {rel }}(\mathcal{W W} \rightarrow \mathcal{A A})=\frac{19 g_{\mathrm{DC}}^{4}(n-1)}{144 \pi M_{\mathcal{W}}^{2}} \\
\sigma v_{\mathrm{rel}}(\mathcal{W W} \rightarrow \mathcal{A} s)=\frac{2 g_{\mathrm{DC}}^{4}(n-1)}{9 \pi n M_{\mathcal{W}}^{2}} \\
\sigma v_{\mathrm{rel}}(\mathcal{W W} \rightarrow s s)=\frac{11 g_{\mathrm{DC}}^{4}}{144 \pi n M_{\mathcal{W}}^{2}} .
\end{gathered}
$$

When $\mathrm{SO}(\mathcal{N}-1)$ confines, we need to distinguish two cases:

- For even $\mathcal{N}$ roughly all $\mathcal{W}$ 's end up in 1-baryons. The final DM abundance is set by $\mathcal{B B}$ annihilations, as they have a large cross section, of order $\sigma_{\mathrm{ann}} \sim 1 / \Lambda_{\mathrm{DC}}^{2}$, giving $Y_{\mathrm{DM}} \sim \min \left(M_{\mathcal{W}} / \alpha_{\mathrm{DC}}^{2}, \Lambda_{\mathrm{DC}}\right) / M_{\mathrm{Pl}}$ for particles with mass of order $M_{\mathcal{W}}+\Lambda_{\mathrm{DC}}$.

- For odd $\mathcal{N}$ roughly all $\mathcal{W}$ 's end up in 2-baryons that decay to 0-baryons, so that the $\mathcal{W}$ abundance negligibly contributes to the final DM abundance, approximated by $Y_{\mathrm{DM}} \sim \Lambda_{\mathrm{DC}} / M_{\mathrm{Pl}}$ for particles with mass of order $\Lambda_{\mathrm{DC}}$. Given that in this case the cross-section $\sigma_{\text {ann }} \approx 1 / \Lambda_{\mathrm{DC}}^{2}$ cannot be extrapolated from a perturbative calculation, the overall coefficient has to be assumed.

The relic abundance is plotted in figure 3 as a function of $\Lambda_{\mathrm{DC}}$ and $M_{\mathcal{W}}$. Notice that, since DM never contains more than one heavy constituent, the relevant annihilation crosssection is always taken to be of order $1 / \Lambda_{\mathrm{DC}}^{2}$. Again, the correct DM abundance is obtained for masses of order $100 \mathrm{TeV}$ or heavier. 


\subsubsection{DM indirect detection}

The cross section for $\mathcal{B B}$ annihilations, that gives indirect detection signals, is

$$
\sigma_{\mathcal{B B}} \sim \mathcal{O}(1) / \Lambda_{\mathrm{DC}}^{2}
$$

where the $\mathcal{O}(1)$ factor is $\sim 100$ in the QCD proton case. DM annihilations produce dark glue-balls, that decay into SM particles. The HESS bound is plotted in figure 3 as a black line, assuming that the dark baryons constitute the totality of DM. Also in this case the region where DM is a thermal relic is not probed.

\subsubsection{DM direct detection}

For even $\mathcal{N}, \mathrm{DM}$ contains one heavy $\mathcal{W}$ constituent and the direct detection cross section is

$$
\sigma_{\mathrm{SI}}=\left(\frac{M_{\mathcal{B}}}{w}\right)^{2} \frac{m_{N}^{4} f^{2}}{4 \pi v^{2}} \sin ^{2} 2 \gamma\left(\frac{1}{M_{S_{1}}^{2}}-\frac{1}{M_{S_{2}}^{2}}\right)^{2} \quad \text { for } \operatorname{SO}(\mathcal{N}) \text { with even } \mathcal{N} .
$$

with $M_{\mathcal{B}} \simeq M_{\mathcal{W}}$.

For odd $\mathcal{N}, \mathrm{DM}$ is a glue-ball that contains zero $\mathcal{W}$ constituents ('odd-ball'), and its direct detection is qualitatively different. The odd-ball coupling to $s$ - that mixes with the Higgs - can be computed by extending soft theorems. One-loop RGE running for $E<M_{\mathcal{W}}<E_{0}$ can be written as

$$
\frac{1}{\alpha_{\mathrm{DC}}(E)}=\frac{1}{\alpha_{\mathrm{DC}}\left(E_{0}\right)}-\frac{b_{H}}{2 \pi} \ln \frac{E}{M_{\mathcal{W}}}-\frac{b_{G}}{2 \pi} \ln \frac{M_{\mathcal{W}}}{E_{0}}
$$

where $b_{H}=-22(\mathcal{N}-3) / 3$ in the broken $\operatorname{SO}(\mathcal{N}-1)$ theory, and $b_{G}=(45-22 \mathcal{N}) / 3=$ $b_{H}+b_{\mathcal{W}}$ in the unbroken theory, with $b_{\mathcal{W}}=-7$. From this we compute how, at fixed highenergy value of the gauge coupling, $\alpha_{\mathrm{DC}}\left(E_{0}\right)$, the scale $E=\Lambda_{\mathrm{DC}}$ at which $\alpha_{\mathrm{DC}}$ becomes non-perturbative depends on $M_{\mathcal{W}}$ and thereby $s$, finding:

$$
\frac{s}{\Lambda_{\mathrm{DC}}} \frac{\partial \Lambda_{\mathrm{DC}}}{\partial s}=-\frac{b_{\mathcal{W}}}{b_{H}}=-\frac{21}{22(\mathcal{N}-3)}=-\frac{7 \alpha_{\mathrm{DC}}}{2 \pi} \ln \frac{M_{\mathcal{W}}}{\Lambda_{\mathrm{DC}}} .
$$

Then the odd-ball (or glue-ball) mass term $M_{\mathcal{B}}^{2} \mathcal{B}^{2} / 2$ with $M_{\mathcal{B}} \propto \Lambda_{\mathrm{DC}}$ gets promoted to ${ }^{11}$

$$
\mathscr{L}=\frac{M_{\mathcal{B}}^{2}}{2} \mathcal{B}^{2}\left(1-\frac{2 b_{\mathcal{W}}}{b_{H}} \frac{s}{w}+\cdots\right) .
$$

The resulting direct detection cross section is

$$
\sigma_{\mathrm{SI}}=\left(\frac{b_{\mathcal{W}}}{2 b_{H}} \frac{M_{\mathcal{B}}}{w}\right)^{2} \frac{m_{N}^{4} f^{2}}{4 \pi v^{2}} \sin ^{2} 2 \gamma\left(\frac{1}{M_{S_{1}}^{2}}-\frac{1}{M_{S_{2}}^{2}}\right)^{2} \quad \text { for } \operatorname{SO}(\mathcal{N}) \text { with odd } \mathcal{N} .
$$

Figure 3 shows the final results. Direct detection could probe a small region where DM is a thermal relic.

\footnotetext{
${ }^{11}$ The factor in parenthesis can be rewritten in terms of $\alpha_{\mathrm{DC}}$ : this computation is equivalent to using the usual Higgs soft theorem of eq. (2.14) (that becomes $-\left(\mathcal{A}_{\mu \nu}^{a}\right)^{2} 7 s \alpha_{\mathrm{DC}} / 8 \pi w$ with SO factors) and using the scale anomaly to compute the baryonic matrix element of such operator.
} 


\subsubsection{Special cases}

To conclude, we mention some special cases.

For $\mathcal{N}=3$ one has $\mathrm{SO}(3) \rightarrow \mathrm{SO}(2)$, which is the same as $\mathrm{SU}(2) \rightarrow \mathrm{U}(1)$ with a scalar in the adjoint. The U(1) does not confine, leaving long range interactions among DM particles. Furthermore, topological defects are possible. The viability of such models will be discussed in a separate publication.

For $\mathcal{N}=4$ the identity $\mathrm{SO}(4) \cong \mathrm{SU}(2)^{2}$ holds and the vector $\mathcal{W}$ lies in the adjoint 3 of the unbroken $\mathrm{SO}(3)$.

For $\mathcal{N}=6$ an extra model with $\mathcal{S}$ in the spinorial of $\mathrm{SO}(6)$ breaks univocally to $\mathrm{SU}(3)$ leaving the scalon as only scalar; in view of the identity $\mathrm{SO}(6) \cong \mathrm{SU}(4)$ this model has been already discussed as $\mathrm{SU}(4)$.

For $\mathcal{N}=8$, the group $\mathrm{SO}(8)$ has three representations with dimension 8 (the fundamental and two spinorials) related by a $S_{3}$ outer automorphism: all 8 representations are real and break $\mathrm{SO}(8)$ to $\mathrm{SO}(7)$.

\section{A fundamental of $\operatorname{Sp}(\mathcal{N})$}

The group $\operatorname{Sp}(\mathcal{N})$ is defined for even $\mathcal{N}$ as the transformations that leave invariant the tensor $\gamma_{\mathcal{N}} \equiv \mathbb{I}_{\mathcal{N} / 2} \otimes \epsilon$, where $\epsilon_{i j}$ is the 2-dimensional anti-symmetric tensor. The fundamental representation of $\operatorname{Sp}(\mathcal{N})$ is pseudo-real. We thereby introduce a complex $\mathcal{N}$-dimensional scalar $\mathcal{S}$. The Lagrangian, given in eq. (1.1), conserves an accidental baryon $\mathrm{U}(1)$, by virtue of $\mathcal{S}^{T} \gamma \mathcal{S}=0$. The adjoint is the trace-less symmetric representation with dimension $\mathcal{N}(\mathcal{N}+1) / 2$. The RGE are

$$
\begin{aligned}
& (4 \pi)^{2} \frac{d g_{\mathrm{DC}}}{d \ln \mu}=-\frac{11 \mathcal{N}+21}{6} g_{\mathrm{DC}}^{3} \\
& (4 \pi)^{2} \frac{d \lambda_{\mathcal{S}}}{d \ln \mu}=\frac{3}{16}(\mathcal{N}+4) g_{\mathrm{DC}}^{4}-3(\mathcal{N}+1) g_{\mathrm{DC}}^{2} \lambda_{\mathcal{S}}+4(4+\mathcal{N}) \lambda_{\mathcal{S}}^{2}
\end{aligned}
$$

\subsection{Sp: Higgs phase}

Again the RGE gives that $\mathcal{S}$ can radiatively acquire a vacuum expectation value as in eq. (2.1), breaking the gauge group $\operatorname{Sp}(\mathcal{N})$ to $\operatorname{Sp}(\mathcal{N}-2)$. At the same time, the accidental $\mathrm{U}(1)$ global dark-baryon number gets rotated to an unbroken global $\mathrm{U}(1)$ with generator

$$
\operatorname{diag}(1, \ldots, 1,1,1)+\operatorname{diag}(0, \ldots, 0,1,-1)=\operatorname{diag}(1, \ldots, 1,2,0)
$$

obtained combining the original $\mathrm{U}(1)$ with the diagonal generator of the broken $\mathrm{Sp}(2)$. Writing the gauge bosons as

$$
T^{a} \mathcal{G}_{\mu}^{a}=\frac{1}{2}\left(\begin{array}{c|cc}
\mathcal{A}_{\mu} & \mathcal{X}_{\mu}^{*} & \gamma_{\mathcal{N}-2} \mathcal{X}_{\mu} \\
\hline \mathcal{X}_{\mu} & \mathcal{Z}_{\mu} & \sqrt{2} \mathcal{W}_{\mu} \\
\gamma_{\mathcal{N}-2} \mathcal{X}^{*} & -\sqrt{2} \mathcal{W}_{\mu}^{*} & -\mathcal{Z}_{\mu}
\end{array}\right)
$$


the perturbative spectrum is:

- the scalon singlet $s$;

- one real $\mathcal{Z}$ with mass $M_{\mathcal{Z}}=g_{\mathrm{DC}} w / 2$ and zero dark baryon charge;

- one complex $\mathcal{W}$, with mass $\mathcal{M}_{\mathcal{W}}=M_{\mathcal{Z}}$ and dark baryon charge 2 .

For $\mathcal{N}=2$ this is the $\mathrm{Sp}(2)=\mathrm{SU}(2)$ model of [7-9] where $\mathcal{W}$ and $\mathcal{Z}$ are co-stable DM candidates. For $\mathcal{N} \geq 4$ the spectrum contains extra particles:

- $\mathcal{N}-2$ complex massive vectors $\mathcal{X}$ in the fundamental representation of $\operatorname{Sp}(\mathcal{N}-2)$ with mass $M_{\mathcal{X}}=M_{\mathcal{W}} / \sqrt{2}$ and dark baryon charge 1 ;

- the massless vectors $\mathcal{A}$ of $\operatorname{Sp}(\mathcal{N}-2)$.

The $\mathcal{Z}$ boson decays into $\mathcal{A}$ 's. At perturbative level the $\mathcal{W}$ and $\mathcal{X}$ are DM candidates, co-stable thanks to accidental baryon number conservation. The cubic vector vertices are

$\mathcal{Z W}^{*} \mathcal{W}, \quad \mathcal{A} \mathcal{A} \mathcal{A}, \quad \mathcal{A} \mathcal{X} \mathcal{X}^{*}, \quad \mathcal{X} \mathcal{X W}^{*}, \quad \mathcal{X X}^{*} \mathcal{Z}$.

Condensation of $\operatorname{Sp}(\mathcal{N}-2)$. When the theory becomes strongly coupled, $\operatorname{Sp}(\mathcal{N}-2)$ confines giving the following spectrum of asymptotic states:

- The scalon $s$, the $\mathcal{Z}$ and $\mathcal{W}$ bosons, and dark glue-balls $\mathcal{A A}$.

- Two kinds of dark mesons: the unstable $\mathcal{X}^{\dagger} \mathcal{X}$ and $\mathcal{X}^{\dagger} \mathcal{D}_{\mu} \mathcal{X}$, which have the same quantum numbers as $s$ and $\mathcal{Z}$, and $\mathcal{M}_{\mu}=\mathcal{X}^{T} \gamma_{\mathcal{N}-2} \mathcal{D}_{\mu} \mathcal{X}$, with dark baryon number 2 as $\mathcal{W}$. Only one linear combination of $\mathcal{M}$ and $\mathcal{W}$ appears among the stable asymptotic states, while the other corresponds to a resonance. A similar situation holds for $s$ and $\mathcal{X}^{\dagger} \mathcal{X}$, and for $\mathcal{Z}$ and $\mathcal{X}^{\dagger} \mathcal{D} \mathcal{X}$.

- Dark baryons $\mathcal{B}$ (defined as states formed with one $\epsilon_{i_{1} \cdots i_{\mathcal{N}-2}}$ tensor) are not stable because the $\epsilon$ tensor can be decomposed as $\epsilon_{i_{1} \cdots i_{\mathcal{N}-2}}=\gamma_{i_{1} i_{2}} \cdots \gamma_{i_{\mathcal{N}-3} i_{\mathcal{N}-2}}+$ permutations [37]. This means that $\mathcal{B}$ splits into $\mathcal{N} / 2-1$ mesons $\mathcal{M}$.

Both the $\mathcal{W}$ and the mesons $\mathcal{M}$ carry charge 2 under conserved $\mathrm{U}(1)$ baryon number.

\subsection{Sp: condensed phase}

Confinement of $\operatorname{Sp}(\mathcal{N})$ gives rise to the following bound states:

- $\mathcal{S}^{\dagger} \mathcal{S}$ corresponding to $s$ and $\mathcal{X}^{\dagger} \mathcal{X}$ (not distinguished by any quantum number);

- $\mathcal{S}^{\dagger} \mathcal{D}_{\mu} \mathcal{S}$ corresponding to $\mathcal{Z}_{\mu}$ and $\mathcal{X}^{\dagger} \mathcal{D}_{\mu} \mathcal{X}$;

- $\mathcal{S}^{T} \gamma_{\mathcal{N}} \mathcal{D}_{\mu} \mathcal{S}$ corresponding to $\mathcal{W}_{\mu}$ and $\mathcal{X}^{T} \gamma_{\mathcal{N}-2} \mathcal{D}_{\mu} \mathcal{X}$

- dark glue-balls $\mathcal{G G}$, corresponding to $\mathcal{A A}$.

The condensed phase coincides with the Higgs phase, in agreement with our generalization of the Fradkin-Shenker theorem. 


\subsection{Sp: phenomenology}

\subsubsection{Relic DM abundance}

At large $\mathcal{N}$ the dominant perturbative annihilation cross-section is

$$
\sigma v_{\text {rel }}\left(\mathcal{X X} \mathcal{X}^{*} \rightarrow \mathcal{A A}\right)=\frac{19 C_{\text {fund }}\left(4 C_{\text {fund }}-C_{\text {adj }}\right) g_{\text {DC }}^{4}}{288 \pi d_{\text {fund }} M_{\mathcal{X}}^{2}}
$$

with $d_{\text {fund }}=2(\mathcal{N}-2), C_{\text {fund }}=(\mathcal{N}-1) / 4, C_{\text {adj }}=\mathcal{N} / 2$. Annihilation and semi-annihilation cross sections of the $V=\mathcal{W}, \mathcal{Z}$ vectors into $s s$ and $V s$ are as in the $\mathrm{SU}(2)$ model [7].

As $\mathcal{X}$ annihilate more than $\mathcal{W}$, the latter can have a larger relic abundance. The two sectors $(\mathcal{W}, \mathcal{Z}$ and $\mathcal{X}, \mathcal{A})$ are however coupled by $\mathcal{A} \mathcal{W} \leftrightarrow \mathcal{X X}$ processes, that thermalize their relative abundances, so that the lighter $\mathcal{X}$ would get a larger abundance than the heavier $\mathcal{W}$. The final abundance depends on which process decouples earlier: a detailed computation would be needed.

When $\operatorname{Sp}(\mathcal{N}-2)$ condenses, about half $\mathcal{X}$ form stable mesons $\mathcal{M}$. The cubic vertex $\mathcal{X} \mathcal{X W}^{*}$ becomes a $\mathcal{M W}$ mass mixing. In the limit $\Lambda_{\mathrm{DC}} \ll w$ their masses are $M_{\mathcal{M}}=\sqrt{2} M_{\mathcal{W}}$ so that $\mathcal{M}$ decays to $\mathcal{W}$ and glue-balls (before that $\mathcal{M M}^{*}$ annihilations with $\sigma_{\text {ann }} \sim \pi R_{B}^{2}$ deplete the $\mathcal{M}$ abundance), leaving $\mathcal{W}$ as the DM candidate. We estimate that the final DM relic density is approximated by perturbative freeze-out abundance of $\mathcal{W}$ (up to the suppression present if glue-balls decay slowly when they dominate the energy density). Hence, differently from the previous cases, in these models dark glue-balls can be so light that they can be probed by collider experiments, for example by measuring Higgs properties.

\subsubsection{Indirect detection}

The indirect detection cross section is given by the perturbative expressions (2.10) with $n=1$, given that the $\mathcal{W}$ is neutral under the unbroken $\operatorname{Sp}(\mathcal{N}-2)$, analogously to the $\mathrm{SU}(2)$ model. The final results are shown in figure 4 (left panel) for $\mathcal{N}=4$. Since the relic density is mainly governed by the perturbative freeze-out of $\mathcal{W}$, the parameter space where DM is a thermal relic can be probed by future experiments.

\subsubsection{Direct detection}

The direct detection cross section is as in the $\mathrm{SU}(2)$ model [7], and the region where DM is thermally produced is fully allowed, as shown in the left panel of figure 4 and will be marginally probed in the future.

\section{A fundamental of $G_{2}$}

We consider $G_{2}$ because it is the only exceptional group that is broken by its fundamental in an unique way, leaving the scalon and no extra scalars, such that the Higgs phase is expected to be equivalent to the condensed phase. $G_{2}$ has 14 generators and a real fundamental with dimension 7 . The invariant tensors of $G_{2}$ are $\delta_{i j}, \epsilon_{i_{1} \cdots i_{7}}$, and $O_{i j k}$, the anti-symmetric tensor that defines octonion multiplication $e_{i} e_{j}=-\delta_{i j}+O_{i j k} e_{k}$ [38]. The 
$\operatorname{Sp}(4)$

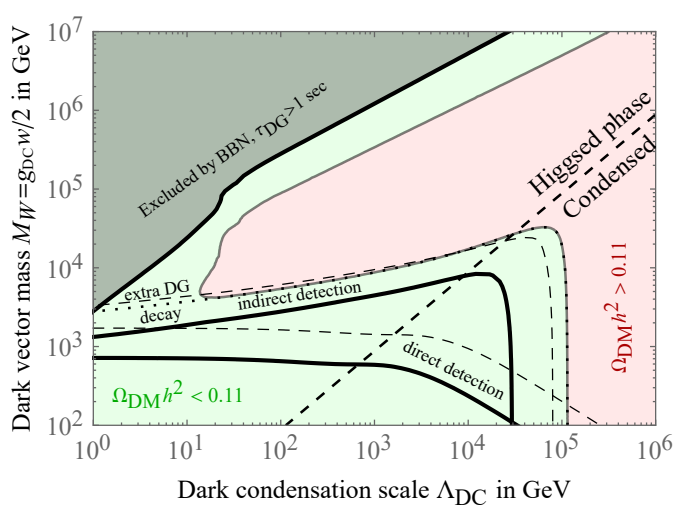

$G_{2}$

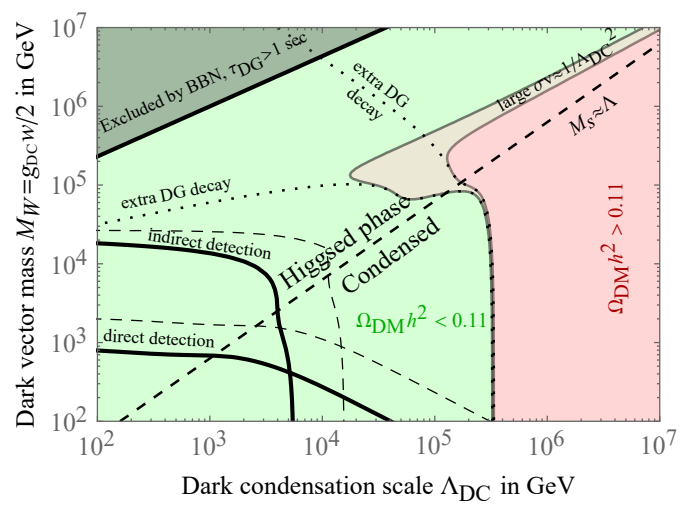

Figure 4. As in figure 1, for $\operatorname{Sp}(\mathcal{N})$ (left) and $G_{2}$ models (right).

most generic renormalizable Lagrangian with a real scalar $\mathcal{S}_{i}$ in the fundamental has one non-vanishing quartic $(\mathcal{S} \cdot \mathcal{S})^{2}$, while $\mathcal{S}^{3}$ cubic interactions vanish. Such Lagrangian enjoys a $\mathcal{S} \rightarrow-\mathcal{S}$ accidental symmetry.

Normalizing $G_{2}$ generators in the fundamental as $\operatorname{Tr}\left(T^{a} T^{b}\right)=\delta^{a b}$, and generators in the adjoint as $\operatorname{Tr}\left(T^{a} T^{b}\right)=4 \delta^{a b}$, the RGE are

$$
(4 \pi)^{2} \frac{d g_{\mathrm{DC}}}{d \ln \mu}=-\frac{29}{2} g_{\mathrm{DC}}^{3}, \quad(4 \pi)^{2} \frac{d \lambda_{\mathcal{S}}}{d \ln \mu}=4 g_{\mathrm{DC}}^{4}-24 g_{\mathrm{DC}}^{2} \lambda_{\mathcal{S}}+30 \lambda_{\mathcal{S}}^{2} .
$$

\section{$5.1 G_{2}$ : Higgs phase}

A vacuum expectation value of $\mathcal{S}$ breaks $G_{2} \rightarrow \mathrm{SU}(3)$. The $G_{2}$ adjoint decomposes as $14=8 \oplus 3 \oplus \overline{3}=\mathcal{A} \oplus \mathcal{W} \oplus \overline{\mathcal{W}}$ under $\mathrm{SU}(3)$. See appendix A.4 for the explicit embedding of the $\mathrm{SU}(3)$ subalgebra into $G_{2}$. The perturbative spectrum is:

- the scalon $s$;

- the 8 massless vectors $\mathcal{A}$ in the adjoint of $\mathrm{SU}(3)$;

- vectors $\mathcal{W}$ with mass $M_{\mathcal{W}}^{2}=g_{\mathrm{DC}}^{2} w^{2} / 3$ in the $3 \oplus \overline{3}$ of $\mathrm{SU}(3)$.

Notice that complex $\mathcal{W}$ 's emerge from a real theory. It is useful to compare $G_{2}$ to $\mathrm{SU}(4)$, that has 15 generators and 8 scalar degrees of freedom in its complex fundamental 4 . The $G_{2} \rightarrow \mathrm{SU}(3)$ theory differs from the $\mathrm{SU}(4) \rightarrow \mathrm{SU}(3)$ theory because of the absence of the $\mathcal{Z}$ vector and because of the presence of $\epsilon^{\alpha \beta \gamma} \mathcal{W}_{\alpha} \mathcal{W}_{\beta} \mathcal{W}_{\gamma}$ gauge interactions.

Condensation of SU(3). Taking into account the confinement of SU(3) gives the following singlets:

- mesons $\mathcal{M}=\mathcal{W}_{\alpha} \overline{\mathcal{W}}^{\alpha}$ which decay to glue-balls;

- $\mathcal{A A}$ glue-balls;

- baryons $\epsilon^{\alpha \beta \gamma} \mathcal{W}_{\alpha} \mathcal{W}_{\beta} \mathcal{W}_{\gamma}$ and $\epsilon_{\alpha \beta \gamma} \overline{\mathcal{W}}^{\alpha} \overline{\mathcal{W}}^{\beta} \overline{\mathcal{W}}^{\gamma}$ constructed contracting with the $\mathrm{SU}(3)$ anti-symmetric tensor $\epsilon$. 
The $\mathcal{W W W}$ and the $\overline{\mathcal{W W W}}$ decay to the same final state, in such a way that the decay amplitude cancels for an appropriate combination $\operatorname{Re} \mathcal{W} \mathcal{W} \mathcal{W}=(\mathcal{W W} \mathcal{W}+\overline{\mathcal{W W W}}) / \sqrt{2}$, similarly to what happens for neutral kaons (one combination is long lived, one combination is short lived). Stability can be understood in terms of the charge conjugation symmetry $\mathrm{C}$ of the Lagrangian:

$$
\mathcal{W}_{\alpha} \rightarrow-\overline{\mathcal{W}}^{\alpha}, \quad \mathcal{A}_{\text {imag }} \rightarrow \mathcal{A}_{\text {imag }}, \quad \mathcal{A}_{\text {real }} \rightarrow-\mathcal{A}_{\text {real }},
$$

where $\mathcal{A}_{\text {real }}\left(\mathcal{A}_{\text {imag }}\right)$ are the $\mathrm{SU}(3)$ vectors with real (imaginary) generators. Interactions dictated by $\mathrm{SU}(3)$ gauge invariance respect this symmetry because it reduces (up to a phase) to the usual $\mathrm{SU}(3)$ complex conjugation; one can check that this symmetry is respected also by the extra $\mathcal{W} \mathcal{W} \mathcal{W}, A \mathcal{W} \mathcal{W}$ interactions. Then, the $\operatorname{Re} \mathcal{W} \mathcal{W}$ baryon is stable being odd under the $\mathrm{C}$ symmetry, while $\mathcal{W W W}-\overline{\mathcal{W W W}}$ is C-even and decays through the $\mathcal{W W W}$ interactions.

\section{$5.2 \quad G_{2}:$ condensed phase}

In the confined $G_{2}$-invariant phase, the spectrum is described by

- $\mathcal{M}=\mathcal{S}_{i} \mathcal{S}_{i}$ mesons which decay in glue-balls;

- $\mathcal{G G}$ glue-balls;

- $O_{i j k} \mathcal{S}_{i} \mathcal{S}_{j} \mathcal{S}_{k}$ baryons built with the rank-3 invariant antisymmetric tensor $O$ and with derivatives (not shown);

- $\epsilon_{i j k l m n s} \mathcal{S}_{i} \mathcal{G}_{j k} \mathcal{G}_{l m} \mathcal{G}_{n s}$ baryons built with the $\epsilon$ invariant antisymmetric tensor. The $G_{2}$ vectors $\mathcal{G}_{i j}=T_{i j}^{a} \mathcal{G}^{a}$ are anti-symmetric in $i j$ (like SO vectors) and do not fill the most generic anti-symmetric matrix (unlike $\mathrm{SO}$ vectors).

The two baryons have the same spin and mix [39]. In the broken theory they are comparably heavy because $\mathcal{G G} \mathcal{G}$ gets its $\mathcal{A W} \mathcal{W}^{*}$ component. The lightest baryon is stable, because of the $\mathcal{S} \rightarrow-\mathcal{S}$ accidental symmetry.

The symmetry that remains unbroken in the Higgs phase corresponds, in the condensed phase, to $\mathcal{S} \rightarrow \eta \mathcal{S}$ where $\eta=\operatorname{diag}(-1,-1,-1,1,1,1,1)$ in the basis given in appendix A.4. This flips the $3+\overline{3}$ indices and leaves invariant the $3-\overline{3}$ indices as well as $\mathcal{S}_{7}$. This component gets a vacuum expectation value in our basis, so that this symmetry remains unbroken in the Higgs phase. This symmetry acts on $G_{2}$ vectors as $\mathcal{G}_{i j} \rightarrow(\eta \mathcal{G} \eta)_{i j}$ i.e.

$$
\mathcal{G}^{1,3,4,6,8,10,11,13} \rightarrow-\mathcal{G}^{1,3,4,6,8,10,11,13}, \quad \mathcal{G}^{2,5,7,9,12,14} \rightarrow \mathcal{G}^{2,5,7,9,12,14},
$$

which agrees with eq. (5.2). The compatibility of this symmetry with the Lie algebra is checked as follows: $f_{a b c}=0$ if one or three indices correspond to odd generators. This symmetry is an inner automorphism of the real group $G_{2}$ that when restricted to its $\mathrm{SU}(3)$ subgroup acts as complex conjugation, which is the outer automorphism of $\mathrm{SU}(3)$ that exchanges $3 \leftrightarrow-\overline{3}$. 
Both $O \mathcal{S S S}$ and $\epsilon \mathcal{S G G G}$ baryons are odd under $\eta$, because both $\epsilon_{i_{1} \cdots i_{7}}$ and $O_{i j k}$ contain an odd number of indices from the set $\{1,2,3\}$. Indeed, the only nonzero elements of the octonion algebra are

$$
O_{123}=O_{516}=O_{624}=O_{435}=O_{471}=O_{673}=O_{572}=1
$$

up to entries obtained by antisymmetry. Therefore, the two baryon structures have the same quantum numbers and mix into the physical stable baryon. To establish the correspondence of the baryons let us consider the Goldstone part of the baryon $\operatorname{Re}\left(\epsilon_{\alpha \beta \gamma} \mathcal{W}_{\alpha} \mathcal{W}_{\beta} \mathcal{W}_{\gamma}\right)$ in the Higgs phase. For instance

$$
\operatorname{Re}\left(\mathcal{W}_{1} \mathcal{W}_{2} \mathcal{W}_{3}\right) \sim \operatorname{Re}\left(\frac{\mathcal{S}_{1}+i \mathcal{S}_{4}}{\sqrt{2}} \frac{\mathcal{S}_{2}+i \mathcal{S}_{5}}{\sqrt{2}} \frac{\mathcal{S}_{3}+i \mathcal{S}_{6}}{\sqrt{2}}\right)=\frac{\mathcal{S}_{1} \mathcal{S}_{2} \mathcal{S}_{3}-\mathcal{S}_{1} \mathcal{S}_{5} \mathcal{S}_{6}-\mathcal{S}_{4} \mathcal{S}_{2} \mathcal{S}_{6}-\mathcal{S}_{4} \mathcal{S}_{5} \mathcal{S}_{3}}{2 \sqrt{2}}
$$

coincides with the Goldstone part of $O_{i j k} \mathcal{S}_{i} \mathcal{S}_{j} \mathcal{S}_{k}$, by virtue of eq. (5.4).

In conclusion, the same spectrum is obtained in the Higgs and condensed phases of a $G_{2}$ gauge theory with a scalar in its fundamental. The equivalence is more sophisticated because of the breaking of a real group to a complex subgroup:

- the meson $\mathcal{S}^{T} \mathcal{S}$ corresponds to the scalon $s$

- the operator $\mathcal{S}^{T} \mathcal{D}_{\mu} \mathcal{S}$ does not give rise to a $\mathcal{Z}_{\mu}$ due to the anti-symmetry of the generators (see footnote 8);

- dark glue-balls in the condensed phase correspond to dark glue-balls in the Higgs phase;

- the lightest baryon, admixture of $\mathcal{S} \mathcal{S}$ and $\mathcal{S G G G}$, corresponds to the baryon $\operatorname{Re} \mathcal{W W} \mathcal{W}$ of the Higgs phase;

- the C-even baryon $\operatorname{Im} \mathcal{W} \mathcal{W} \mathcal{W}$, that mixes with the scalon and with $\mathrm{SU}(3)$ glue-balls, corresponds to resonances of the mesons and glueballs of $G_{2}$.

\section{$5.3 \quad G_{2}:$ phenomenology}

The theory is similar to the $\mathrm{SU}(4) \rightarrow \mathrm{SU}(3)$ theory, up to the absence of the $\mathcal{Z}$ boson and to the presence of $\mathcal{W W W}$ interactions. The perturbative $\mathcal{W} \mathcal{W}^{*} \rightarrow \mathcal{A A}, \mathcal{A} s$, ss DM annihilation cross sections are thereby equal to those given in eq. (2.9). Furthermore there are extra $\mathcal{W W} \rightarrow \mathcal{W}^{*}$ semi-annihilations, as in DM models with an ad-hoc $\mathbb{Z}_{3}$ symmetry [40]. The perturbative $\mathcal{W}$ relic density is thereby similar to the density in the $\mathrm{SU}(4) \rightarrow \mathrm{SU}(3)$ model. $\mathcal{W W W}$ interactions give an extra difference at non-perturbative level: when the $\mathcal{W W W}$ and $\overline{\mathcal{W W W}}$ baryons form, only half of them survive in the stable C-odd component, analogously to a $K^{0}$ beam after the decay of the short-lived $K_{S}^{0}$. As DM is now real, indirect detection is enhanced by a order one factor, while direct detection is as in the $\mathrm{SU}(4) \rightarrow \mathrm{SU}(3)$ model (after taking into account the slightly different RGE and thereby scalon mass). Figure 4 (right) summarizes our final results. 


\section{Conclusions}

We have studied models with a new dark gauge group $\mathcal{G}$ and a new dark scalar $\mathcal{S}$, selected such that the Higgs phase (where $\mathcal{S}$ gets a vacuum expectation value, breaking $\mathcal{G}$ to a sub-group $\mathcal{H}$ ) is dual to the confined phase (where $\mathcal{G}$ gets strongly interacting). Fradkin, Shenker and others proved that this happens for $\mathcal{G}=\mathrm{SU}(2)$ with a scalar $\mathcal{S}$ in its fundamental. We argued that the correspondence of the two phases holds whenever the scalar $\mathcal{S}$ breaks $\mathcal{G}$ to a unique sub-group $\mathcal{H} .{ }^{12}$ In these cases $\mathcal{S}$ admits a single quartic self-coupling, and the broken theory contains a single Higgs scalar, that we call $s$. This happens when $\mathcal{S}$ fills a fundamental of the $\mathrm{SU}(\mathcal{N}), \operatorname{SO}(\mathcal{N}), \operatorname{Sp}(\mathcal{N}), G_{2}$ groups. Table 1 summarizes how the Higgs/confinement duality is realized in each model.

We studied such models from the point of view of DM phenomenology. When presenting final results, we further restricted the parameter space assuming that:

- the cosmological DM abundance is reproduced thermally;

- the $\mathcal{G} \rightarrow \mathcal{H}$ symmetry breaking occurs dynamically à la Coleman-Weinberg;

- the $\mathcal{S}$ vacuum expectation value also induces the observed Higgs mass.

Thanks to these extra assumptions, DM phenomenology is described by one free parameter, the dark gauge coupling $g_{\mathrm{DC}}$ of $\mathcal{G}$. The confined phase is obtained smoothly for $g_{\mathrm{DC}} \sim$ $4 \pi / \sqrt{\mathcal{N}}$. Smaller perturbative $g_{\mathrm{DC}}$ correspond to the Higgs phase. As strong interactions (either of $\mathcal{G}$ or $\mathcal{H}$ ) are often involved, cosmology often selects the DM mass typical of strong interactions: about $100 \mathrm{TeV}$. Such DM is heavy enough that the considered models are experimentally allowed. Of course, some of the above assumptions can be relaxed, giving more general phenomenology. The various DM candidates are listed in table 2 for each case, together with their main features.

Our main results can be summarized as follows:

- In section 2 we considered $\mathcal{G}=\mathrm{SU}(\mathcal{N})$ with $\mathcal{S}$ in its complex fundamental representation. In both phases the theory admits an unbroken accidental U(1) dark-baryon number, leading to DM stability. DM is the baryon made by $\mathcal{N}$ scalars $\mathcal{S}$. In the Higgs phase $\mathcal{G}$ is broken to $\mathcal{H}=\mathrm{SU}(\mathcal{N}-1)$, and one of the $\mathcal{N}$ scalars gets replaced by its vacuum expectation value $\langle\mathcal{S}\rangle$, so that DM is made by $\mathcal{N}-1$ heavy vectors. $\mathcal{H}$ confines at a lower scale $\Lambda_{\mathrm{DC}}$, giving a strong suppression of the cosmological DM relic density. Being made by heavy constituents, the size of DM (and thereby its cross sections in cosmology and in indirect detection) is set by its Bohr-like radius. Higgs soft theorems allowed to compute DM direct detection. Figure 1 summarizes DM phenomenology, showing that all experimental bounds are satisfied. Furthermore, DM is accompanied by lighter, unstable dark glue-balls that can potentially be probed by their coupling to the Higgs.

\footnotetext{
${ }^{12}$ Indeed, in case the sub-group $\mathcal{H}$ were not unique, there would be different spectra of asymptotic states associated to each possible breaking. Hence, the condensed phase of $\mathcal{G}$, which is presumably unique since it is dominated by gauge interactions, cannot be equivalent to the Higgs phases.
} 


\begin{tabular}{|c|c|c|}
\hline Group & Higgs phase & Condensed phase \\
\hline $\mathrm{SU}(\mathcal{N}) \rightarrow \mathrm{SU}(\mathcal{N}-1)$ & $\begin{array}{c}s \\
\mathcal{Z}_{\mu} \\
\epsilon_{\mathcal{N}-1} \mathcal{W}^{\mathcal{N}-1} \\
\mathcal{A} \mathcal{A}\end{array}$ & $\begin{array}{c}\mathcal{S}^{\dagger} \mathcal{S} \\
\mathcal{S}^{\dagger} \mathcal{D}_{\mu} \mathcal{S} \\
\epsilon_{\mathcal{N}} \mathcal{S}^{\mathcal{N}} \\
\mathcal{G} \mathcal{G}\end{array}$ \\
\hline $\mathrm{SO}(\mathcal{N}) \rightarrow \mathrm{SO}(\mathcal{N}-1)$ & $\begin{array}{c}{ }^{s} \\
\epsilon_{\mathcal{N}-1} \mathcal{A} \ldots \mathcal{A} \quad(\text { for odd } \mathcal{N}) \\
\epsilon_{\mathcal{N}-1} \mathcal{W} \mathcal{A} \ldots \mathcal{A} \quad(\text { for even } \mathcal{N}) \\
\mathcal{A} \mathcal{A}\end{array}$ & 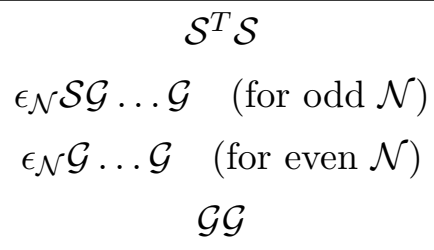 \\
\hline $\operatorname{Sp}(\mathcal{N}) \rightarrow \operatorname{Sp}(\mathcal{N}-2)$ & $\begin{array}{c}s, \mathcal{X}^{\dagger} \mathcal{X} \\
\mathcal{Z}_{\mu}, \mathcal{X}^{\dagger} \mathcal{D}_{\mu} \mathcal{X} \\
\mathcal{W}_{\mu}, \mathcal{X}^{T} \gamma_{\mathcal{N}-2} \mathcal{D}_{\mu} \mathcal{X} \\
\mathcal{A} \mathcal{A}\end{array}$ & $\begin{array}{c}\mathcal{S}^{\dagger} \mathcal{S} \\
\mathcal{S}^{\dagger} \mathcal{D}_{\mu} \mathcal{S} \\
\mathcal{S}^{T} \gamma_{\mathcal{N}} \mathcal{D}_{\mu} \mathcal{S} \\
\mathcal{G G}\end{array}$ \\
\hline$G_{2} \rightarrow \mathrm{SU}(3)$ & $\begin{array}{c}s \\
\operatorname{Re} \mathcal{W W W} \\
\mathcal{A A}\end{array}$ & $\begin{array}{c}\mathcal{S}^{T} \mathcal{S} \\
\mathcal{S} \mathcal{S}, \mathcal{S} \mathcal{G} \mathcal{G} \mathcal{G} \\
\mathcal{G G}\end{array}$ \\
\hline
\end{tabular}

Table 1. Correspondence of the asymptotic states between the Higgs and confined phases.

\begin{tabular}{|c|c|l|c|}
\hline Group & Global symmetry & \multicolumn{1}{|c|}{ DM candidate } & DM Annihilation \\
\hline $\mathrm{SU}(\mathcal{N})$ & Dark baryon number & Baryon $\epsilon S^{\mathcal{N}} \cong \mathcal{W}^{n}$ & Bohr-like $-1 / \Lambda_{\mathrm{DC}}^{2}$ \\
$\mathrm{SO}\left(\mathcal{N}_{\text {even }}\right)$ & O-parity & 1-ball $\epsilon \mathcal{G}^{\mathcal{N} / 2} \cong \mathcal{W} \mathcal{A}^{(n-1) / 2}$ & $1 / \Lambda_{\mathrm{DC}}^{2}$ \\
$\mathrm{SO}\left(\mathcal{N}_{\text {odd }}\right)$ & O-parity & 0 -ball $\epsilon \mathcal{S} \mathcal{G}^{(\mathcal{N}-1) / 2 \cong \mathcal{A}^{n / 2}}$ & $1 / \Lambda_{\mathrm{DC}}^{2}$ \\
$\mathrm{Sp}(\mathcal{N})$ & Dark baryon number & Meson $\mathcal{S} \gamma \mathcal{S} \cong \mathcal{W}, \mathcal{X X}$ & Perturbative \\
$G_{2}$ & Inner automorphism & Baryon $\epsilon \mathcal{S} \mathcal{G}^{3}, O \mathcal{S}^{3} \cong \operatorname{Re} \mathcal{W}^{3}$ & Bohr-like $-1 / \Lambda_{\mathrm{DC}}^{2}$ \\
\hline
\end{tabular}

Table 2. Dark matter candidates, thier stabilizing symmetries, and dominant annihilation mechanisms in the various models.

- In section 3 we considered $\mathcal{G}=\mathrm{SO}(\mathcal{N})$ with $\mathcal{S}$ in its real fundamental representation. $\mathrm{DM}$ is stable because of O-parity, a symmetry related to SO groups analogous to how parity is related to the rotation group. For odd $\mathcal{N}, \mathrm{DM}$ is the baryon made by one heavy scalar $\mathcal{S}$. For even $\mathcal{N}, \mathrm{DM}$ is an odd dark glue-ball containing no scalars $\mathcal{S}$. In the Higgs phase $\mathcal{G}$ is broken to $\mathcal{H}=\operatorname{SO}(\mathcal{N}-1)$, that confines at a lower scale $\Lambda_{\mathrm{DC}}$, giving a strong suppression of the cosmological DM relic density. Since $\mathrm{DM}$ contains light dark gluons, it has a larger size set by $1 / \Lambda_{\mathrm{DC}}$. An extension of Higgs soft theorems allowed to compute direct detection of odd-ball DM. Figure 3 summarizes DM phenomenology, showing that all experimental bounds are satisfied. 
- In section 4 we considered $\mathcal{G}=\operatorname{Sp}(\mathcal{N})$ with $\mathcal{S}$ in its pseudo-real fundamental. DM is stable thanks to an accidental $\mathrm{U}(1)$ dark baryon number. In the Higgs phase $\mathcal{G}$ is broken to $\mathcal{H}=\operatorname{Sp}(\mathcal{N}-2)$, giving two co-stable vector DM candidates $\mathcal{W}$ (neutral under $\mathcal{H}$ and with dark baryon number 2) and $\mathcal{X}$ (charged under $\mathcal{H}$ and with dark baryon number 1 ), with masses $M_{\mathcal{X}}=M_{\mathcal{W}} / \sqrt{2}$. When $\mathcal{H}$ confines at a lower scale $\Lambda_{\mathrm{DC}}$, two $\mathcal{X}$ 's form a meson and their cosmological DM relic density gets strongly suppressed. DM remains as $\mathcal{W}$ with cosmological relic density approximately not suppressed by $\mathcal{H}$ confinement. Because of this, dark glue-balls can be especially light in Sp models. Up to the presence of dark glue-balls, DM phenomenology is similar to the $\mathrm{SU}(2)=\mathrm{Sp}(2)$ model. Figure 4 (left) summarizes DM phenomenology, showing that all experimental bounds are satisfied.

- In section 5 we considered the exceptional group $G_{2}$ with $\mathcal{S}$ in its real fundamental. In the confined phase, the $\mathcal{S S S}$ and $\mathcal{S G G G}$ baryons remain stable thanks to an accidental $\mathcal{S} \rightarrow-\mathcal{S}$ symmetry. In the Higgs phase $G_{2}$ is broken to $\mathcal{H}=\mathrm{SU}(3)$ and the theory contains massive $\mathcal{W} \oplus \overline{\mathcal{W}}$ vectors in the $3 \oplus \overline{3}$. The theory contains $\mathcal{W} \mathcal{W} \mathcal{W}$ gauge interaction characteristic of $G_{2}$, which give $\mathcal{W W} \rightarrow \mathcal{W}^{*}$ processes. As a result the $\operatorname{Im} \mathcal{W W W}$ baryon decays, while the $\operatorname{Re} \mathcal{W W W}$ remains as a stable DM candidate, thanks to an inner automorphism of $G_{2}$ that reduces to charge conjugation of SU(3). Stability arises as a quantum mechanical interference phenomenon, analogous to how the neutral kaons split into long-lived and short-lived eigenstates. DM size is set by the Bohr-like radius. Figure 4 (right) summarizes DM phenomenology, showing that all experimental bounds are satisfied.

As we sometimes relied on approximations, various aspects of each model can be more precisely computed. Furthermore, it will be interesting to see if other choices of scalar representations that do not satisfy the Higgs/confinement duality lead to DM candidates with distinct phenomenology.

\section{Acknowledgments}

This work was supported by the ERC grant NEO-NAT, by MIUR under contract number 2017L5W2PT, and by the INFN grant FLAVOR. We thank Claudio Bonati, Christian Gross, Thomas Hambye, Paolo Panci, Michele Redi, and Filippo Sala for discussions. 


\section{A Generators}

For completeness we provide here the $\mathrm{SU}(\mathcal{N}), \operatorname{SO}(\mathcal{N}), \operatorname{Sp}(\mathcal{N})$ and $G_{2}$ generators in the fundamental representation.

\section{A.1 $\mathrm{SU}(\mathcal{N})$}

The $\mathrm{SU}(\mathcal{N})$ generators are given in terms of a generalization of the Pauli matrices $\sigma_{a} / 2$ :

$$
\begin{aligned}
& \left(T_{\alpha \beta}^{(1)}\right)_{\gamma \delta}=\frac{1}{2}\left(\delta_{\alpha \gamma} \delta_{\beta \delta}+\delta_{\alpha \delta} \delta_{\beta \gamma}\right) \quad(1 \leq \alpha<\beta \leq \mathcal{N}) \\
& \left(T_{\alpha \beta}^{(2)}\right)_{\gamma \delta}=-\frac{i}{2}\left(\delta_{\alpha \gamma} \delta_{\beta \delta}-\delta_{\alpha \delta} \delta_{\beta \gamma}\right) \quad(1 \leq \alpha<\beta \leq \mathcal{N}) \\
& \left(T_{\alpha}^{(3)}\right)_{\gamma \delta}=\left\{\begin{array}{cl}
\frac{1}{\sqrt{2 \alpha(\alpha-1)}} \delta_{\gamma \delta} & (\gamma<\alpha) \\
-\sqrt{\frac{\alpha-1}{2 \alpha}} \delta_{\gamma \delta} & (\gamma=\alpha \\
0 & (\gamma>\alpha)
\end{array}\right.
\end{aligned}
$$

Altogether they are $\frac{1}{2} \mathcal{N}(\mathcal{N}-1)+\frac{1}{2} \mathcal{N}(\mathcal{N}-1)+(\mathcal{N}-1)=\mathcal{N}^{2}-1$ generators, which can be collected as

$$
\begin{array}{llllrl}
T^{1} & =T_{12}^{(1)}, & T^{2}=T_{12}^{(2)}, & T^{3}=T_{2}^{(3)}, & T^{4}=T_{13}^{(1)}, & T^{5}=T_{13}^{(2)}, \\
T^{6}=T_{23}^{(1)}, & T^{7}=T_{23}^{(2)}, & T^{8}=T_{3}^{(3)}, & \ldots & T^{\mathcal{N}^{2}-1}=T_{\mathcal{N}}^{(3)},
\end{array}
$$

with normalization $\operatorname{Tr}\left(T^{a} T^{b}\right)=\frac{1}{2} \delta^{a b}$.

\section{A.2 $\mathrm{SO}(\mathcal{N})$}

The $\frac{1}{2} \mathcal{N}(\mathcal{N}-1)$ generators of $\mathrm{SO}(\mathcal{N})$ are given in terms of the $T_{\alpha \beta}^{(2)} \mathrm{SU}$ generators defined in eq. (A.1b) as

$$
T^{1}=2 T_{12}^{(2)}, \quad T^{2}=2 T_{13}^{(2)}, \quad \ldots \quad T^{\mathcal{N}-1}=2 T_{1 \mathcal{N}}^{(2)}, \quad \ldots \quad T^{\frac{1}{2} \mathcal{N}(\mathcal{N}-1)}=2 T_{\mathcal{N}-1, \mathcal{N}}^{(2)}
$$

with normalization $\operatorname{Tr}\left(T^{a} T^{b}\right)=2 \delta^{a b}$.

\section{A.3 $\operatorname{Sp}(\mathcal{N})$}

Symplectic Lie groups exist for even $\mathcal{N}=2 \ell$. The $\ell(2 \ell+1)$ generators of $\operatorname{Sp}(2 \ell)$ can be written in terms of the $\ell$-dimensional SU generators $T_{\alpha \beta}^{(1)}$ and $T_{\alpha \beta}^{(2)}$ defined in eq. (A.1) as

$$
\frac{1}{\sqrt{2}} T_{\alpha \beta}^{(2)} \otimes \mathbb{I}_{2}, \quad \frac{1}{\sqrt{2}} T_{\alpha \beta}^{(1)} \otimes \sigma_{k}, \quad \frac{1}{2} T_{\alpha \alpha}^{(1)} \otimes \sigma_{k},
$$

for $1 \leq \alpha<\beta \leq \ell$ and $k=1,2,3$. In fact, these are $\frac{1}{2} \ell(\ell-1)+\frac{1}{2} \ell(\ell-1) \cdot 3+\ell \cdot 3=\ell(2 \ell+1)$ elements, which can be collected as

$$
\begin{aligned}
T^{1} & =\frac{1}{\sqrt{2}} T_{12}^{(2)} \otimes \mathbb{1}_{2} & \ldots & T^{\frac{1}{2} \ell(\ell-1)} & =\frac{1}{\sqrt{2}} T_{\ell-1, \ell}^{(2)} \otimes \mathbb{1}_{2} \\
T^{\frac{1}{2} \ell(\ell-1)+1} & =\frac{1}{\sqrt{2}} T_{12}^{(1)} \otimes \sigma_{1} & \ldots & T^{2 \ell(\ell-1)} & =\frac{1}{\sqrt{2}} T_{\ell-1, \ell}^{(1)} \otimes \sigma_{3} \\
T^{2 \ell(\ell-1)+1} & =\frac{1}{2} T_{11}^{(1)} \otimes \sigma_{1} & \ldots & T^{\ell(2 \ell+1)} & =\frac{1}{2} T_{\ell \ell}^{(1)} \otimes \sigma_{3},
\end{aligned}
$$

with normalization $\operatorname{Tr}\left(T^{a} T^{b}\right)=\frac{1}{2} \delta^{a b}$. The invariant tensor $\gamma_{\mathcal{N}} \equiv \mathbb{I}_{\mathcal{N} / 2} \otimes i \sigma_{2}$ satisfies $\left(T^{a}\right)^{T} \gamma_{\mathcal{N}}+\gamma_{\mathcal{N}} T^{a}=0$ 


\section{$\mathrm{A} .4 G_{2}$}

$G_{2}$ has 14 generators which can be written in terms of the 7-dimensional matrices $T_{\alpha \beta}^{(2)}$ defined in eq. (A.1b) as [41]

$$
\begin{aligned}
T^{1} & =T_{51}^{(2)}-T_{24}^{(2)} & T^{8} & =\frac{1}{\sqrt{3}}\left(T_{24}^{(2)}+T_{51}^{(2)}-2 T_{73}^{(2)}\right) \\
T^{2} & =T_{54}^{(2)}-T_{12}^{(2)} & T^{9} & =-\frac{1}{\sqrt{3}}\left(T_{54}^{(2)}+T_{12}^{(2)}-2 T_{67}^{(2)}\right) \\
T^{3} & =T_{25}^{(2)}-T_{14}^{(2)} & T^{10} & =\frac{1}{\sqrt{3}}\left(T_{14}^{(2)}+T_{25}^{(2)}-2 T_{36}^{(2)}\right) \\
T^{4} & =T_{43}^{(2)}-T_{16}^{(2)} & T^{11} & =\frac{1}{\sqrt{3}}\left(T_{16}^{(2)}+T_{43}^{(2)}-2 T_{72}^{(2)}\right) \\
T^{5} & =T_{31}^{(2)}-T_{46}^{(2)} & T^{12} & =\frac{1}{\sqrt{3}}\left(T_{46}^{(2)}+T_{31}^{(2)}-2 T_{57}^{(2)}\right) \\
T^{6} & =T_{62}^{(2)}-T_{35}^{(2)} & T^{13} & =\frac{1}{\sqrt{3}}\left(T_{35}^{(2)}+T_{62}^{(2)}-2 T_{71}^{(2)}\right) \\
T^{7} & =T_{65}^{(2)}-T_{23}^{(2)} & T^{14} & =-\frac{1}{\sqrt{3}}\left(T_{65}^{(2)}+T_{23}^{(2)}-2 T_{47}^{(2)}\right),
\end{aligned}
$$

with normalization $\operatorname{Tr}\left(T^{a} T^{b}\right)=\delta^{a b}$. The adjoint decomposes under $\mathrm{SU}(3)$ as $14=8 \oplus 3 \oplus$ $\overline{3}=\mathcal{A} \oplus \mathcal{W} \oplus \overline{\mathcal{W}}$. Among the 7 dimensions, the first three correspond to the embedding of $3 \oplus \overline{3}$, the second three to $3-\overline{3}$, and the 7 th one to the singlet direction. The $\mathrm{SU}(3)$ subalgebra is spanned by $\left\{T^{1}, \ldots, T^{7},-T^{10}\right\}$, with the $\mathrm{SU}(3)$ adjoint vectors embedded as $\mathcal{A}_{1, \ldots, 7}=\mathcal{G}_{1, \ldots, 7}, \mathcal{A}_{8}=-\mathcal{G}_{10}$. The $\mathcal{W}$ are embedded as

$$
\mathcal{W}+\overline{\mathcal{W}}=\left(\mathcal{G}_{8}, \mathcal{G}_{11}, \mathcal{G}_{13}\right), \quad i(\mathcal{W}-\overline{\mathcal{W}})=\left(\mathcal{G}_{9},-\mathcal{G}_{12}, \mathcal{G}_{14}\right)
$$

\section{B Feynman rules}

To derive the Feynman rules for the $\mathrm{SU}(\mathcal{N}) \rightarrow \mathrm{SU}(\mathcal{N}-1)$ breaking pattern we decompose the Lagrangian under the unbroken $\mathrm{SU}(\mathcal{N}-1)$ as follows

$$
\begin{aligned}
-\frac{\mathcal{G}_{\mu \nu}^{a} \mathcal{G}^{a \mu \nu}}{4}+\left|\mathcal{D}_{\mu} \mathcal{S}\right|^{2}= & -\frac{1}{4} \mathcal{A}_{\mu \nu}^{a} \mathcal{A}^{a \mu \nu}-\frac{1}{2} \mathcal{W}_{\mu \nu}^{\dagger} \mathcal{W}^{\mu \nu}-\frac{1}{4} \mathcal{Z}_{\mu \nu} \mathcal{Z}^{\mu \nu}-i g_{\mathrm{DC}}\left(\mathcal{W}_{\mu}^{\dagger} T_{\mathcal{N}-1}^{a} \mathcal{W}_{\nu}\right) \mathcal{A}^{a \mu \nu} \\
& +i g_{\mathrm{DC}} f_{\mathcal{N}}^{\mathcal{N}^{2}-1}\left(\left(\partial_{[\mu} \mathcal{W}_{\nu]}^{\dagger}\right) \mathcal{W}^{\nu} \mathcal{Z}^{\mu}-\mathcal{W}_{\nu}^{\dagger}\left(\partial^{[\mu} \mathcal{W}^{\nu]}\right) \mathcal{Z}_{\mu}-\mathcal{W}_{[\mu}^{\dagger} \mathcal{W}_{\nu]} \partial^{\mu} \mathcal{Z}^{\nu}\right) \\
& -\frac{g_{\mathrm{DC}}^{2}}{2} \mathcal{N}\left(\mathcal{W}_{\mu}^{\dagger} \mathcal{W}^{\mu} \mathcal{Z}_{\nu} \mathcal{Z}^{\nu}-\mathcal{W}_{\mu}^{\dagger} \mathcal{W}_{\nu} \mathcal{Z}^{\mu} \mathcal{Z}^{\nu}\right) \\
& -g_{\mathrm{DC}}^{2} f_{\mathcal{N}}^{\mathcal{N}^{2}-1}\left(2 \mathcal{Z}^{\mu} \mathcal{A}_{\mu}^{a}\left(\mathcal{W}_{\nu}^{\dagger} T_{\mathcal{N}-1}^{a} \mathcal{W}^{\nu}\right)-\left(\mathcal{Z}_{\mu} \mathcal{A}_{\nu}^{a}+\mathcal{Z}_{\nu} \mathcal{A}_{\mu}^{a}\right)\left(\mathcal{W}^{\dagger \mu} T_{\mathcal{N}-1}^{a} \mathcal{W}^{\nu}\right)\right) \\
& +\frac{1}{2} \partial_{\mu} s \partial^{\mu} s+M_{\mathcal{W}}^{2}\left(1+\frac{s}{w}\right)^{2} \mathcal{W}_{\mu}^{\dagger} \mathcal{W}^{\mu}+\frac{1}{2} M_{\mathcal{Z}}^{2}\left(1+\frac{s}{w}\right)^{2} \mathcal{Z}_{\mu} \mathcal{Z}^{\mu}+\cdots
\end{aligned}
$$

where $\cdots$ denotes $\mathcal{W} \mathcal{W}^{*} \mathcal{W} \mathcal{W}^{*}$ vertices. We defined $f_{\mathcal{N}}^{\mathcal{N}^{2}-1}=\sqrt{\mathcal{N} /(2(\mathcal{N}-1))}, \mathcal{D}_{\mu}=$ $\partial_{\mu}-i g_{\mathrm{DC}} T_{\mathcal{N}-1}^{a} \mathcal{A}_{\mu}^{a}$ and

$$
\mathcal{W}_{\mu \nu}=\mathcal{D}_{\mu} \mathcal{W}_{\nu}-\mathcal{D}_{\nu} \mathcal{W}_{\mu}, \quad \mathcal{Z}_{\mu \nu}=\partial_{\mu} \mathcal{Z}_{\nu}-\partial_{\nu} \mathcal{Z}_{\mu}, \quad \mathcal{A}_{\mu \nu}^{a}=\partial_{\mu} \mathcal{A}_{\nu}^{a}-\partial_{\nu} \mathcal{A}_{\mu}^{a}+g_{\mathrm{DC}} f_{\mathcal{N}-1}^{a b c} \mathcal{A}_{\mu}^{b} \mathcal{A}_{\nu}^{b}
$$


The Feynman vertices with all momenta $p_{i}$ incoming are:
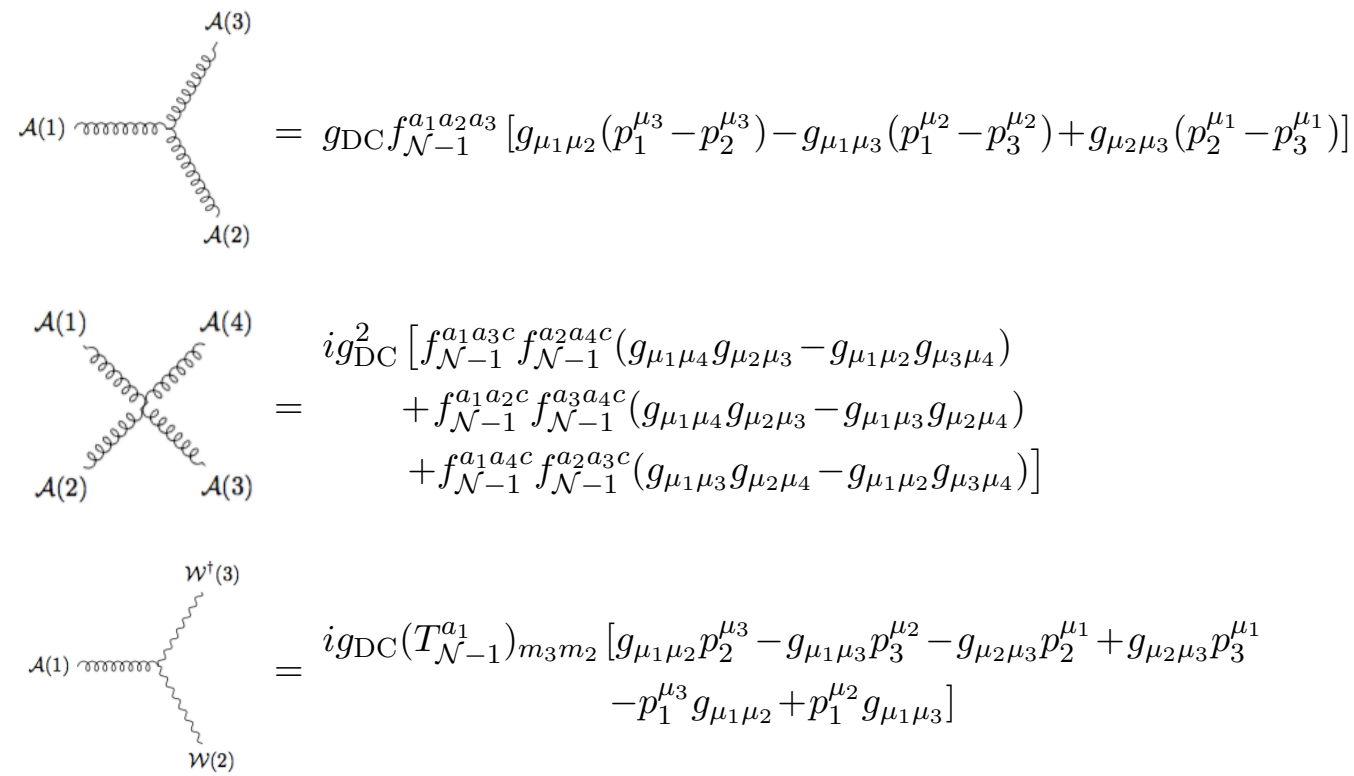

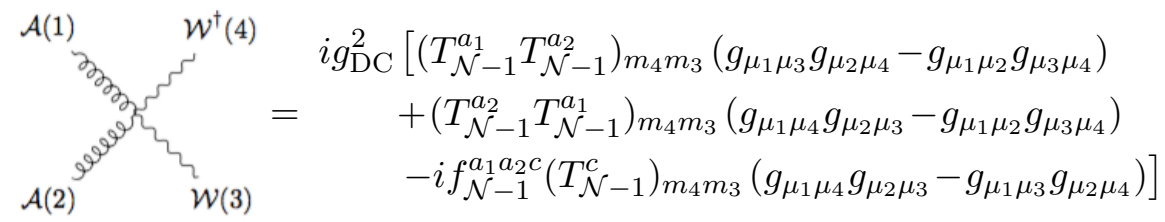
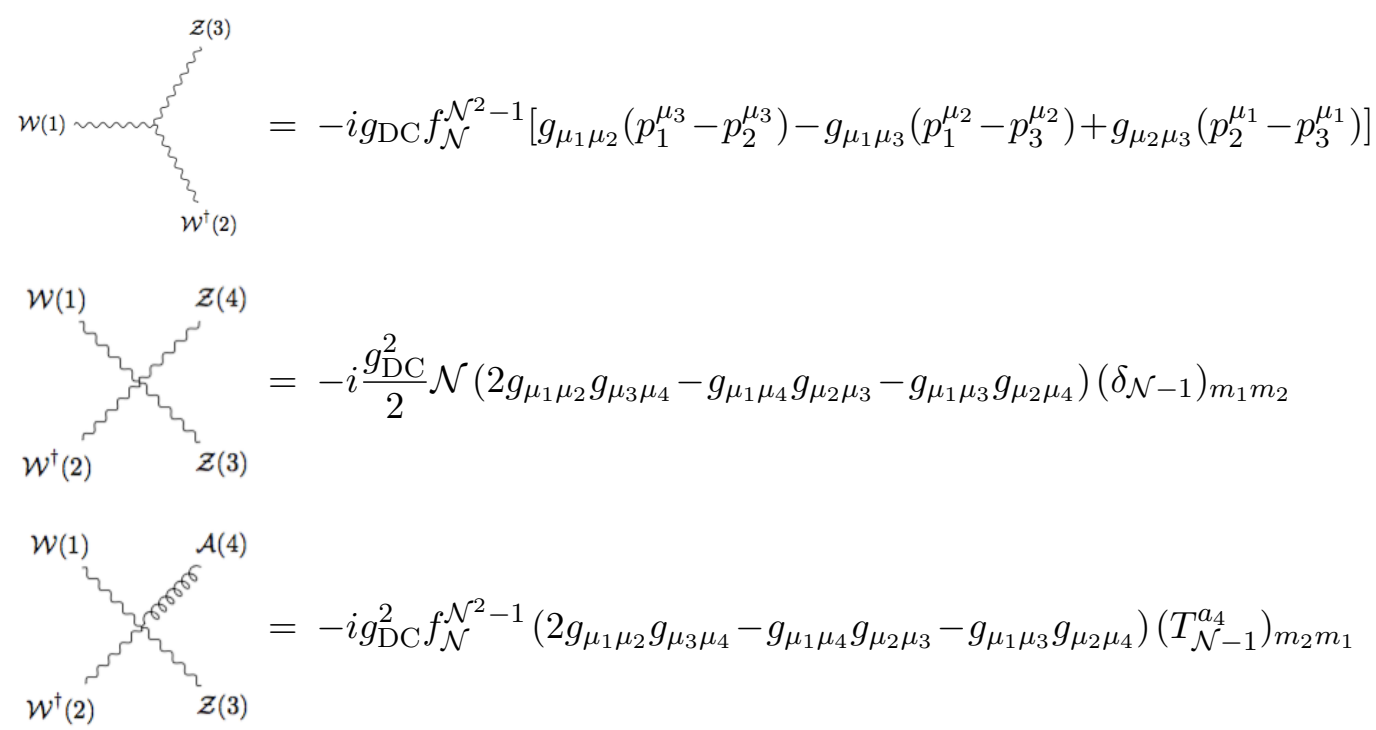

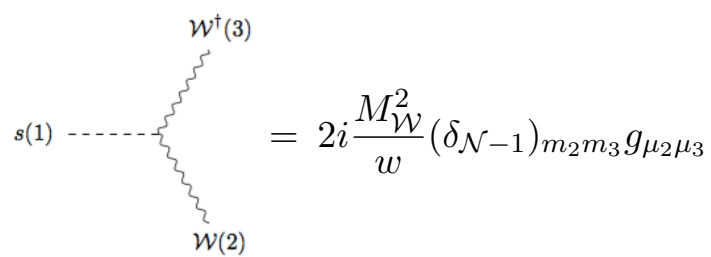



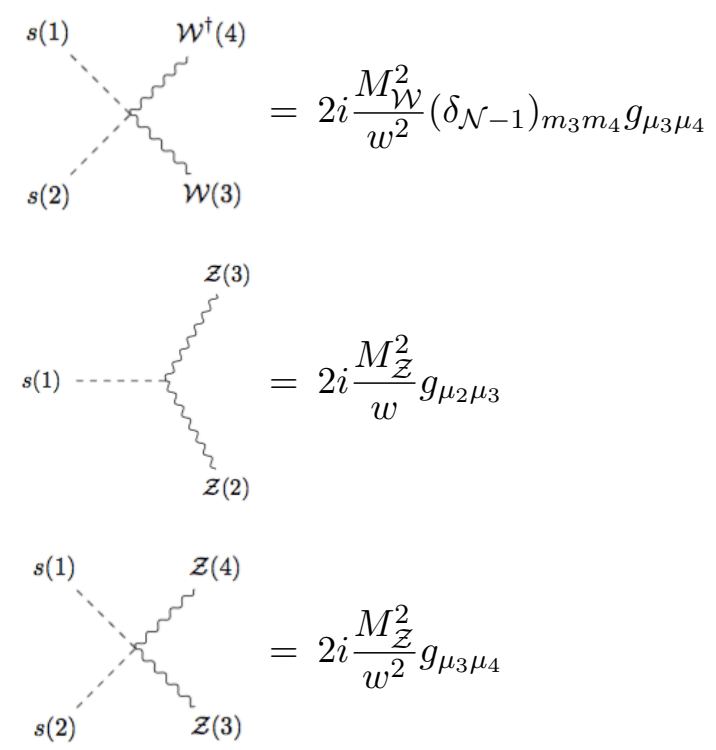

Similar expressions can be derived for the other groups considered in this paper. E.g. the $\mathrm{SO}(\mathcal{N}) \rightarrow \mathrm{SO}(\mathcal{N}-1)$ case is simply obtained from $\mathrm{SU}(\mathcal{N}) \rightarrow \mathrm{SU}(\mathcal{N}-1)$ by dropping vertices involving $\mathcal{Z}$ bosons and taking into account that $\mathcal{W}$ 's become real. The $G_{2}$ group gives extra $\mathcal{W W} \mathcal{W}$ interactions, and Sp gives extra vectors $\mathcal{X}$.

Open Access. This article is distributed under the terms of the Creative Commons Attribution License (CC-BY 4.0), which permits any use, distribution and reproduction in any medium, provided the original author(s) and source are credited.

\section{References}

[1] V. Silveira and A. Zee, Scalar phantoms, Phys. Lett. 161B (1985) 136 [INSPIRE].

[2] C.P. Burgess, M. Pospelov and T. ter Veldhuis, The Minimal model of nonbaryonic dark matter: A Singlet scalar, Nucl. Phys. B 619 (2001) 709 [hep-ph/0011335] [InSPIRE].

[3] M. Farina, D. Pappadopulo and A. Strumia, CDMS stands for Constrained Dark Matter Singlet, Phys. Lett. B 688 (2010) 329 [arXiv:0912.5038] [inSPIRE].

[4] J.M. Cline, K. Kainulainen, P. Scott and C. Weniger, Update on scalar singlet dark matter, Phys. Rev. D 88 (2013) 055025 [Erratum ibid. D 92 (2015) 039906] [arXiv:1306.4710] [INSPIRE].

[5] GAMBIT collaboration, Status of the scalar singlet dark matter model, Eur. Phys. J. C 77 (2017) 568 [arXiv : 1705. 07931] [INSPIRE].

[6] O. Lebedev, H.M. Lee and Y. Mambrini, Vector Higgs-portal dark matter and the invisible Higgs, Phys. Lett. B 707 (2012) 570 [arXiv:1111.4482] [INSPIRE].

[7] T. Hambye, Hidden vector dark matter, JHEP 01 (2009) 028 [arXiv:0811.0172] [InSPIRE].

[8] T. Hambye and M.H.G. Tytgat, Confined hidden vector dark matter, Phys. Lett. B 683 (2010) 39 [arXiv: 0907.1007] [INSPIRE].

[9] T. Hambye and A. Strumia, Dynamical generation of the weak and Dark Matter scale, Phys. Rev. D 88 (2013) 055022 [arXiv: 1306.2329] [INSPIRE]. 
[10] K. Osterwalder and E. Seiler, Gauge Field Theories on the Lattice, Annals Phys. 110 (1978) 440 [INSPIRE].

[11] E.H. Fradkin and S.H. Shenker, Phase Diagrams of Lattice Gauge Theories with Higgs Fields, Phys. Rev. D 19 (1979) 3682 [InSPIRE].

[12] T. Banks and E. Rabinovici, Finite Temperature Behavior of the Lattice Abelian Higgs Model, Nucl. Phys. B 160 (1979) 349 [INSPIRE].

[13] C. Bonati, G. Cossu, A. D'Alessandro, M. D'Elia and A. Di Giacomo, On the phase diagram of the Higgs SU(2) model, PoS (LATTICE2008) 252 (2008) [arXiv:0901.4429] [INSPIRE].

[14] L.F. Abbott and E. Farhi, Are the Weak Interactions Strong?, Phys. Lett. 101B (1981) 69 [INSPIRE].

[15] G. 't Hooft, Topological aspects of quantum chromodynamics, in From the Planck length to the Hubble radius. Proceedings, International School of Subnuclear Physics, Erice, Italy, August 29-September 7, 1998, pp. 216-236 (1998) [hep-th/9812204] [INSPIRE].

[16] A. Trautner, CP and other Symmetries of Symmetries, Ph.D. Thesis, Munich, Tech. U., Universe (2016) [arXiv:1608.05240] [INSPIRE].

[17] C. Gross, O. Lebedev and Y. Mambrini, Non-Abelian gauge fields as dark matter, JHEP 08 (2015) 158 [arXiv : 1505.07480] [INSPIRE].

[18] G. Arcadi, C. Gross, O. Lebedev, Y. Mambrini, S. Pokorski and T. Toma, Multicomponent Dark Matter from Gauge Symmetry, JHEP 12 (2016) 081 [arXiv:1611.00365] [INSPIRE].

[19] O. Antipin, M. Redi, A. Strumia and E. Vigiani, Accidental Composite Dark Matter, JHEP 07 (2015) 039 [arXiv: 1503.08749] [INSPIRE].

[20] A. Mitridate, M. Redi, J. Smirnov and A. Strumia, Dark Matter as a weakly coupled Dark Baryon, JHEP 10 (2017) 210 [arXiv:1707.05380] [INSPIRE].

[21] R. Contino, A. Mitridate, A. Podo and M. Redi, Gluequark Dark Matter, JHEP 02 (2019) 187 [arXiv: 1811.06975] [INSPIRE].

[22] E. Gildener and S. Weinberg, Symmetry Breaking and Scalar Bosons, Phys. Rev. D 13 (1976) 3333 [INSPIRE].

[23] C.J. Morningstar and M.J. Peardon, The Glueball spectrum from an anisotropic lattice study, Phys. Rev. D 60 (1999) 034509 [hep-lat/9901004] [InSPIRE].

[24] S. Dimopoulos, S. Raby and L. Susskind, Light Composite Fermions, Nucl. Phys. B 173 (1980) 208 [INSPIRE].

[25] A. Maas and P. Törek, Predicting the singlet vector channel in a partially broken gauge-Higgs theory, Phys. Rev. D 95 (2017) 014501 [arXiv:1607.05860] [INSPIRE].

[26] A. Maas, R. Sondenheimer and P. Törek, On the observable spectrum of theories with a Brout-Englert-Higgs effect, Annals Phys. 402 (2019) 18 [arXiv:1709.07477] [INSPIRE].

[27] A. Maas and P. Törek, The spectrum of an $\mathrm{SU}(3)$ gauge theory with a fundamental Higgs field, Annals Phys. 397 (2018) 303 [arXiv: 1804.04453] [INSPIRE].

[28] K. Harigaya, M. Ibe, K. Kaneta, W. Nakano and M. Suzuki, Thermal Relic Dark Matter Beyond the Unitarity Limit, JHEP 08 (2016) 151 [arXiv:1606.00159] [INSPIRE].

[29] V. De Luca, A. Mitridate, M. Redi, J. Smirnov and A. Strumia, Colored Dark Matter, Phys. Rev. D 97 (2018) 115024 [arXiv:1801.01135] [InSPIRE]. 
[30] C. Gross, A. Mitridate, M. Redi, J. Smirnov and A. Strumia, Cosmological Abundance of Colored Relics, Phys. Rev. D 99 (2019) 016024 [arXiv: 1811.08418] [InSPIRE].

[31] XENON collaboration, Dark Matter Search Results from a One Ton-Year Exposure of XENON1T, Phys. Rev. Lett. 121 (2018) 111302 [arXiv:1805.12562] [INSPIRE].

[32] Fermi-LAT collaboration, Searching for Dark Matter Annihilation from Milky Way Dwarf Spheroidal Galaxies with Six Years of Fermi Large Area Telescope Data, Phys. Rev. Lett. 115 (2015) 231301 [arXiv: 1503.02641] [INSPIRE].

[33] D. Buttazzo, F. Sala and A. Tesi, Singlet-like Higgs bosons at present and future colliders, JHEP 11 (2015) 158 [arXiv: 1505. 05488] [INSPIRE].

[34] D. Buttazzo, D. Redigolo, F. Sala and A. Tesi, Fusing Vectors into Scalars at High Energy Lepton Colliders, JHEP 11 (2018) 144 [arXiv:1807.04743] [INSPIRE].

[35] H.E.S.S. collaboration, Search for dark matter annihilations towards the inner Galactic halo from 10 years of observations with H.E.S.S, Phys. Rev. Lett. 117 (2016) 111301 [arXiv: 1607.08142] [INSPIRE].

[36] CTA Consortium collaboration, The Dark Matter Programme of the Cherenkov Telescope Array, PoS (ICRC2017) 921 (2018) [arXiv: 1709. 01483] [INSPIRE].

[37] E. Witten, Current Algebra, Baryons and Quark Confinement, Nucl. Phys. B 223 (1983) 433 [INSPIRE].

[38] J.T. Graves, On a Connection between the General Theory of Normal Couples and the Theory of Complete Quadratic Functions of Two Variables, Phil. Mag. 26 (1845) 315.

[39] K. Holland, P. Minkowski, M. Pepe and U.J. Wiese, Exceptional confinement in $G_{2}$ gauge theory, Nucl. Phys. B 668 (2003) 207 [hep-lat/0302023] [INSPIRE].

[40] F. D'Eramo and J. Thaler, Semi-annihilation of Dark Matter, JHEP 06 (2010) 109 [arXiv: 1003.5912] [INSPIRE].

[41] M. Günaydin and F. Gursey, Quark structure and octonions, J. Math. Phys. 14 (1973) 1651 [INSPIRE]. 